\title{
Involvement of FoxO1 in the effects of follicle- stimulating hormone on inhibition of apoptosis in mouse granulosa cells
}

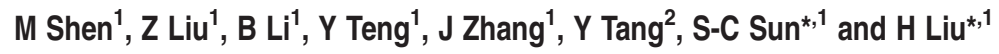

In mammalian ovaries, follicular atresia occurs periodically and destroys almost all the follicles in the ovary. Follicle-stimulating hormone (FSH) acts as the primary survival factor during follicular atresia by preventing apoptosis in granulosa cells. FoxO1 is a critical factor in promoting follicular atresia and granulosa cell apoptosis. FSH inhibits the induction of Fox01. In this report, we investigated the role of FSH-FoxO1 pathway in mouse follicular atresia. FSH dampened stress-induced apoptosis and the expression of FoxO1 and pro-apoptosis genes in mouse granulosa cells (MGCs). In contrast, overexpression of Fox01 inhibited the viability of MGCs and induced the expression of endogenous Fox01. The signaling cascades involved in regulating Fox01 activity upon FSH treatment were identified using FSH signaling antagonists. Blocking protein kinase A (PKA), phosphatidylinositol-3 kinase (PI3K) or protein kinase B (AKT) restored the upregulation of Fox01 and apoptotic signals, which was suppressed by FSH. Moreover, inhibition of PKA or PI3K impaired FSH-induced AKT activity, but inactivation of PI3K or AKT had little effect on PKA activity in the presence of FSH. Correspondingly, constitutive activation of FoxO1 (all three AKT sites were replaced by alanines) also promoted MGC apoptosis despite FSH administration. Furthermore, both luciferase reporter assays and chromatin immunoprecipitation assays showed that Fox01 directly bound to a FoxO-recognized element site within the Fox01 promoter and contributed to the regulation of Fox01 expression in response to FSH. Taken together, we propose a novel model in which FSH downregulates Fox01-dependent apoptosis in MGCs by coordinating the PKA-PI3K-AKT-FoxO1 axis and Fox01Fox01 positive feedback.

Cell Death and Disease (2014) 5, e1475; doi:10.1038/cddis.2014.400; published online 16 October 2014

More than $99 \%$ of mammalian ovarian follicles undergo degeneration during growth and development, a phenomenon known as follicular atresia. ${ }^{1}$ Inappropriate follicular atresia is responsible for certain reproductive disorders, such as polycystic ovarian syndrome and premature ovarian failure (also known as premature menopause), leading to infertility in women. $^{2,3}$ Previous studies have demonstrated a close relationship between follicular atresia and granulosa cell apoptosis in which DNA fragmentation, activation of caspases and upregulation of pro-apoptotic gene expression are seen. ${ }^{4}$ Correspondingly, the maturation of follicles is a complex process that is regulated by gonadotropins and intraovarian regulators. ${ }^{5,6}$ In particular, follicle-stimulating hormone (FSH) is required for the production of estrogen, ${ }^{7}$ growth and development of antral follicles ${ }^{8}$ and the selection of dominant follicles (DFs). ${ }^{9}$ These physiological responses to FSH are achieved by activating several signaling cascades in granulosa cells, including protein kinase $A(P K A)$, protein kinase $B(P K B / A K T)$, p38 mitogen-activated protein kinase
(p38-MAPK) and extracellular signal-regulated kinases 1 and 2 (ERK1/2), which in turn modulate $>100$ different target genes. ${ }^{10}$ The effect of FSH is due to its binding to FSH receptor, which is specifically localized on the plasma membrane of granulosa cells. ${ }^{11} \mathrm{FSH}$ was identified as a major survival factor for antral follicles because of its ability to antagonize apoptosis in granulosa cells. ${ }^{12}$ However, its target genes and the potential mechanism for protection of granulosa cells during this stage remain to be elucidated. ${ }^{13}$

The FoxO subfamily of forkhead transcription factors, which includes FoxO1, FoxO3, FoxO4 and FoxO6, regulates genes required for apoptosis, cell cycle arrest, muscle regeneration, mitophagy, cellular homeostasis, aging and mitochondrial metabolism. ${ }^{14}$ FoxO activity is regulated by numerous posttranslational modifications. When not phosphorylated, FoxO functions as a transcriptional activator or repressor by binding to the FoxO-recognized element (FRE) within the promoters of its target genes. Phosphorylation of FoxO by PKB/AKT in response to insulin, growth factors, hormones and other

\footnotetext{
${ }^{1}$ Department of Animal Genetics, Breeding and Reproduction, College of Animal Science and Technology, Nanjing Agricultural University, Nanjing, China and ${ }^{2}$ Institute of Animal Science, Chinese Academy of Agricultural Science, Beijing, China

*Corresponding author: S-C Sun or H Liu, College of Animal Science and Technology, Nanjing Agricultural University, Weigang 1, Nanjing 210095, China. Tel/Fax: +86 2584399092 (S-CS) or +86 2584395106 (HL); E-mail: sunsc@ @jau.edu.cn or liuhonglin@njau.edu.cn

Abbreviations: AKT, protein kinase B; BSA, bovine serum albumin; ChIP, Chromatin immunoprecipitation; DFs, dominant follicles; DBD, DNA-binding domain; ERK1/2, extracellular signal-regulated kinases 1 and 2 ; ER, estradiol receptor; FSH, follicle-stimulating hormone; FRE, FoxO-recognized element; FasL, Fas ligand; H\&E, hematoxylin and eosin; i.p., intraperitoneal; LH, leutinizing hormone; MGCs, mouse granulosa cells; PBS, phosphate-buffered saline; PI3K, phosphatidylinositol-3 kinase; p38-MAPK, p38 mitogen-activated protein kinases; PKA, protein kinase A; qRT-PCR, quantitative reverse transcription polymerase chain reaction; ROS, reactive oxygen species; SDS, sodium dodecyl sulfate; TBS-T, $50 \mathrm{mM}$ Tris-HCl, pH 7.5, $150 \mathrm{mM} \mathrm{NaCl}, 0.1 \%$ Tween 20; TRAIL, tumor necrosis factor-related apoptosis-inducing ligand; TUNEL, terminal deoxynucleotidyl transferase-mediated dUTP nick end labeling; WT, wild-type

Received 13.6.14; revised 11.8.14; accepted 14.8.14; Edited by A Stephanou
} 
stimuli results in the exclusion of FoxO from the nucleus and subsequent degradation in the cytosol, inhibiting FoxOdependent transcription. ${ }^{15}$ In the absence of insulin and/or growth factors, PKB/AKT suppression induces dephosphorylation and nuclear localization of FoxO, leading to cell cycle arrest and apoptosis via the activation of genes, such as cyclin-dependent kinase inhibitor (p27KIP1), BCL2-like 11 (Bim), tumor necrosis factor-related apoptosis-inducing ligand (TRAIL) and Fas ligand (FasL). ${ }^{16-20}$ Moreover, acetylation and mono-ubiquitination are other ways that FoxO activity is controlled. $^{21}$

A recent study in the ovary suggests a potential function for FoxO1 in follicular development. ${ }^{22}$ An immunohistochemical assay demonstrated the centralization and nuclear distribution of FoxO1 staining in rat granulosa cells from atretic follicles. ${ }^{23}$ FoxO1 inhibits proliferation and steroidogenesis in mouse granulosa cells (MGCs), which may further induce apoptosis and follicular atresia. Conversely, FSH upregulates the expression of genes involved in cell proliferation and estrogen production, promotes follicular growth, and decreases granulosa cell apoptosis. ${ }^{24}$ The opposing activities of FoxO1 and FSH on follicular development and granulosa cell survival imply a reciprocal inhibition between these two factors in the ovary. As a target of $\mathrm{FSH}$ signaling, FoxO1 activity is negatively influenced by $\mathrm{FSH}^{22}$ In granulosa cells, $\mathrm{FSH}$ induces phosphorylation of FoxO1 by $\mathrm{PKB} / \mathrm{AKT}$ at several residues, resulting in retrotranslocation of FoxO1 from the nucleus to the cytoplasm. ${ }^{25}$ Although FoxO1 is abundant in cultured granulosa cells, FSH treatment significantly decreases its expression. 22,24,26,27 Thus, FSH may mediate ovarian function in a FoxO1-dependent manner. ${ }^{23}$ However, few further clues exist regarding the regulation of FoxO1 in response to $\mathrm{FSH}$ signaling.

Our previous work demonstrated a critical role for FoxO1 in driving MGC apoptosis and follicular atresia. ${ }^{4} \mathrm{FSH}$ has the opposite affect by facilitating follicular maturation and granulosa cell proliferation. ${ }^{10}$ Therefore, we were interested in the contribution of FoxO1 to $\mathrm{FSH}$ signaling cascades. As expected, we found that $\mathrm{FSH}$ significantly reduced FoxO1 expression in MGCs both in vivo and in vitro in agreement with previous reports. ${ }^{22,24,26,27}$ Therefore, we hypothesized that downregulation of FoxO1-induced apoptosis may correlate with the action of FSH on granulosa cell survival. In this study, we investigated the response mechanism of FoxO1 to FSHmediated prevention of apoptosis in MGCs. Our results suggested a primary role for FoxO1 inhibition of $\mathrm{FSH}$ induced MGC survival through coordination of the PKAphosphatidylinositol-3 kinase (PI3K)-AKT-FoxO1 axis and FoxO1-FoxO1 positive feedback.

\section{Results}

FSH protected MGCs from apoptosis in dominant ovarian follicles. It is well established that FSH is the primary survival factor for DFs. ${ }^{28} \mathrm{FSH}$ alone promotes antral follicles growth and development into preovulatory follicles, which will maintain anovulation without the stimulation of leutinizing hormone (LH). ${ }^{29} \mathrm{FSH}$ withdraw (coasting) during this stage leads to granulosa cell apoptosis and follicular atresia. ${ }^{30}$ We therefore developed a corresponding FSH treatment protocol to mimic in vivo DFs growth and atresia as shown in Materials and Methods section and Supplementary Figure S1. In brief, the growth of mouse ovarian DFs was induced by intraperitoneal (i.p.) injection with FSH twice daily (12-h intervals) for 2 days at a dose of $10 \mathrm{IU}$ on day 1 and $5 \mathrm{IU}$ on day 2. FSH was then withdrawn for an additional 24 or $48 \mathrm{~h}$ to stimulate physiological follicular atresia in DFs, or injected i.p. (10 IU per mouse) $6 \mathrm{~h}$ before MGC retrieval. At 48, 72 and $96 \mathrm{~h}$ after the first FSH injection, we collected mouse ovaries or MGCs of DFs for tests. Using the terminal deoxynucleotidyl transferase-mediated dUTP nick end labeling (TUNEL) assay, MGC apoptosis was significantly increased after 24 and $48 \mathrm{~h}$ of FSH deprivation (66 and 90-h groups). Specifically, TUNEL-positive staining was concentrated in MGCs within DFs. In contrast, mice primed with FSH $6 \mathrm{~h}$ before FSH withdrawal showed low apoptotic signals in ovarian MGCs (Figure 1a). Using hematoxylin and eosin (H\&E) staining, we detected the effects of FSH on follicular atresia under the same conditions. Consistent with the data from the TUNEL assay, mice in which FSH was withdrawn displayed more atretic follicles (including DFs) in ovaries compared with those supplemented with FSH (Figure 1b).

A previous study suggested the involvement of a mitochondria-dependent pathway in initiating granulosa cell apoptosis. ${ }^{31}$ To investigate the correlation of this pathway with $\mathrm{FSH}$-induced granulosa cell protection, we analyzed the activities of caspases in MGCs and found that upregulation of caspase-3 and caspase-9 (mediates mitochondriadependent apoptosis) activity was blocked by additional injection of FSH during the FSH withdrawal (Figures 1d and e). However, no significant change in caspase-8 activity (responsible for mitochondria-independent apoptosis) was observed in response to $\mathrm{FSH}$ treatment (Figure 1c). Consistent with this, we showed that the mRNA expression of Bim, but not FasL, is regulated by $\mathrm{FSH}^{32}$ These results showed that FSH may prevent ovarian MGC apoptosis by inhibiting the mitochondria-dependent pathway.

FSH reduced FoxO1 expression in ovarian MGCs. The effects of FSH on FoxO1 expression were measured with quantitative reverse transcription polymerase chain reaction (qRT-PCR) and western blotting. We first examined the mRNA levels of FoxO family members in MGCs collected from ovarian DFs $96 \mathrm{~h}$ after the original $\mathrm{FSH}$ injection in mice and found that FoxO1 and FoxO3a were the most abundant FoxO mRNA isoforms in MGCs of DFs (Figure 2a), in agreement with previous observations. ${ }^{33}$ We next evaluated the responsiveness of FoxO1 and FoxO3a to $\mathrm{FSH}$ treatment. FoxO1 mRNA was inhibited by approximately 14-fold by FSH administration during the FSH withdrawal. However, only about a one- to twofold increase in FoxO3a mRNA occurred under the same conditions (Figure 2b). These data suggested that FSH specifically regulated FoxO1 rather than other FoxO mRNAs in MGCs of DFs, and we thus focused our subsequent studies on FoxO1. qRT-PCR analysis indicated that the high level of FoxO1 mRNA in MGCs induced by $\mathrm{FSH}$ deprivation was significantly decreased in mice primed with FSH 6 h before FSH deprivation (Figure 2c). Consistent with this observation, $\mathrm{FSH}$ treatment suppressed 
a
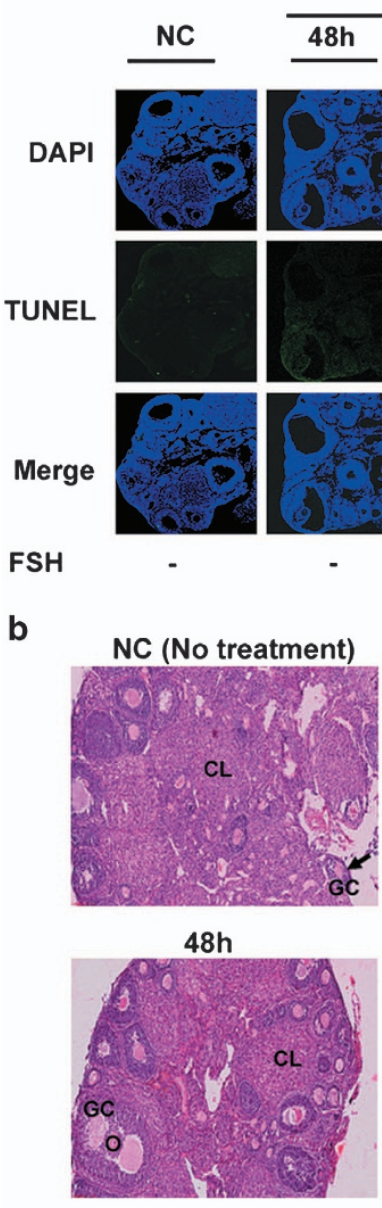

Sample collection time
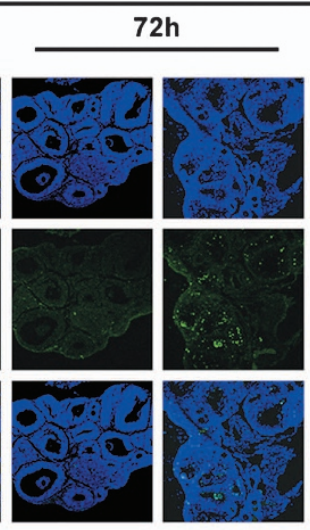

$66 h+$
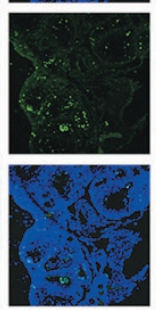

$66 \mathrm{~h}-$
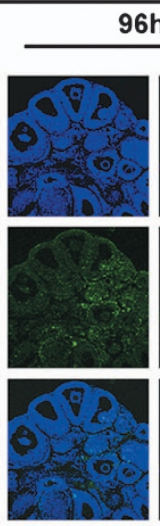

$90 \mathrm{~h}+$
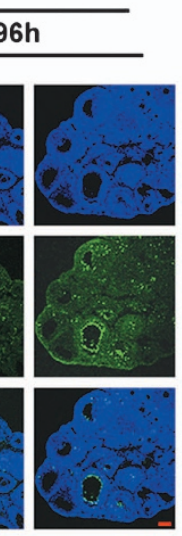

90h-
C
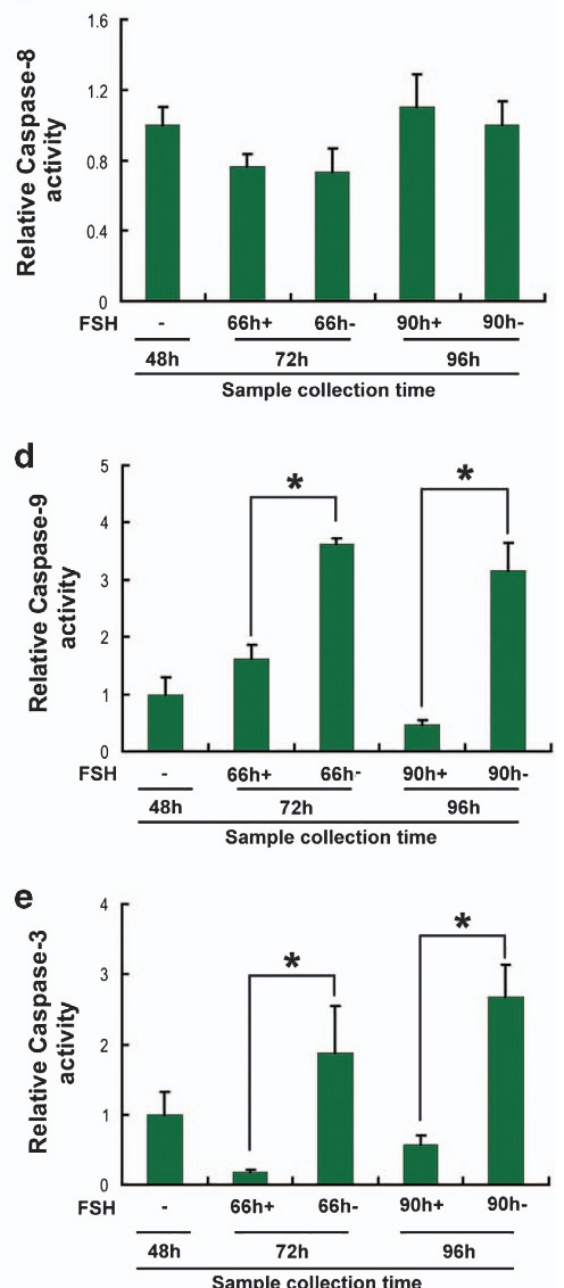

Figure 1 FSH reduced apoptosis in MGCs of ovarian DFs. Mice were injected i.p. with FSH twice daily (12-h intervals, 0800 and 2000 hours) for 2 days at a dose of 10 IU on day 1 and $5 \mathrm{IU}$ on day 2. FSH was then withdrawn for an additional 24 or $48 \mathrm{~h}$, or injected i.p. (10 IU per mouse) $6 \mathrm{~h}$ before MGC collection. (a-e) At 48,72 and $96 \mathrm{~h}$ after the first FSH injection, the right ovaries were paraffin-embedded and serially sectioned for the TUNEL assay (a) and H\&E staining (b). MGCs were isolated from DFs (>200 $\mu \mathrm{m}$ ) in the left ovaries for caspase activity detection (c-e). The data represent the mean \pm S.E. $(n=3)$. ${ }^{*} P<0.05$, Student's $t$-test. Bar, $100 \mu \mathrm{m} .0,00 c y t e ; G C$, granulosa cells; $\mathrm{CL}$, corpus luteum

the expression of FoxO1 protein in MGCs retrieved from DFs during FSH withdrawal (Figures 2d and e). The synchronization of MGC protection and FoxO1 inhibition by $\mathrm{FSH}$ reflects a potential connection between FoxO1 and FSH. In addition, earlier studies had identified FoxO1 as a transactivator of Bim, ${ }^{15,18}$ which is a component of the mitochondriadependent apoptotic pathway ${ }^{34}$ that is also downregulated by $\mathrm{FSH}$ as mentioned above. Therefore, our data raised the possibility that $\mathrm{FSH}$ prevents MGCs from undergoing apoptosis in a FoxO1-dependent manner.

FSH protected MGCs from apoptosis in vitro. Follicular growth and granulosa cell survival are controlled by both ovarian steroids and pituitary gonadotropins. ${ }^{35}$ To further confirm the anti-apoptotic potential of FSH and to rule out the possible actions of other endocrine factors, experiments were performed with cultured granulosa cells harvested from DFs. Exposure to $\mathrm{H}_{2} \mathrm{O}_{2}(200 \mu \mathrm{M})$ induced apoptotic death in about
$85 \%$ of MGCs $6 \mathrm{~h}$ later, and this death was remarkably suppressed by FSH in a dose-dependent manner (Figures 3a and $\mathrm{b}$ ). $\mathrm{FSH}$ at $7.5 \mathrm{IU} / \mathrm{ml}$ tended to show maximal protection and thus was chosen for subsequent experiments. We next studied the time course of the viability of MGCs upon $\mathrm{H}_{2} \mathrm{O}_{2}$ treatment in the presence of $\mathrm{FSH}$. Cells incubated in $200 \mu \mathrm{M}$ $\mathrm{H}_{2} \mathrm{O}_{2}$ for $0,1,2$ or $3 \mathrm{~h}$ were washed with phosphate-buffered saline (PBS) and then cultured in serum-free medium containing $7.5 \mathrm{IU} / \mathrm{ml} \mathrm{FSH}$ for $12 \mathrm{~h}$. Apoptotic signals were indistinguishable from cells with $\mathrm{O}$ h of $\mathrm{H}_{2} \mathrm{O}_{2}$ exposure. $\mathrm{H}_{2} \mathrm{O}_{2}$ incubation for $1 \mathrm{~h}$ led to a marked increase in the rate of TUNEL-positive cells, which was significantly blocked by FSH. However, apoptosis triggered by $>2 \mathrm{~h}$ of $\mathrm{H}_{2} \mathrm{O}_{2}$ exposure could not be rescued by FSH (Figures $3 \mathrm{c}$ and $\mathrm{d}$ and Supplementary Figure S2). Therefore, $1 \mathrm{~h}$ of $\mathrm{H}_{2} \mathrm{O}_{2}$ treatment was considered a 'rescueable' length of time to induce apoptosis in MGCs and was applied in the following experiments. In accordance with the TUNEL assay, qRT-PCR 
a
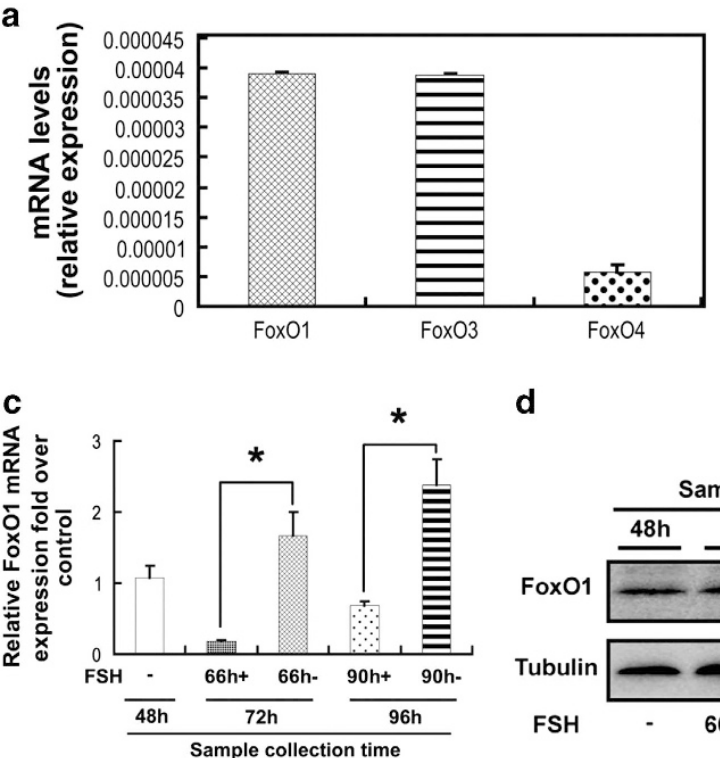

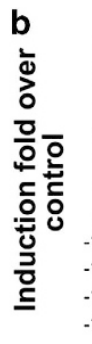

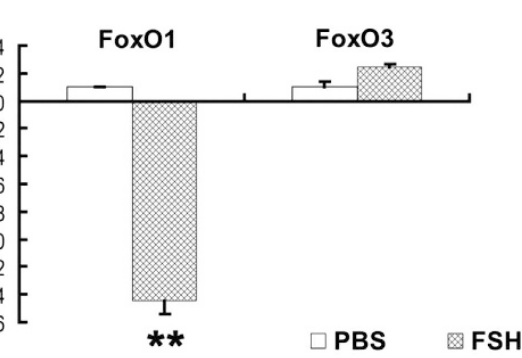

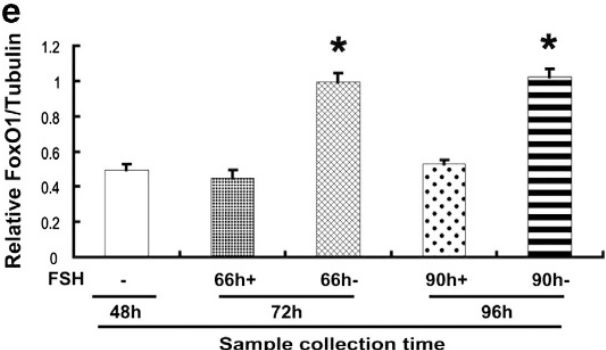

Figure 2 FSH protected follicular MGCs from apoptosis in a FoxO1-dependent manner. Ovarian stimulation and MGC retrieval were performed as described in Supplementary Figure S1. (a) The expression of FoxO family members in MGCs of DFs was measured with qRT-PCR. (b) The responsiveness of FoxO1 and FoxO3a to FSH treatment was detected as above. (c) Transcription changes in FoxO1 in response to FSH-induced MGC protection. (d) The inhibition of FoxO1 expression by FSH was examined using western blotting. $\alpha$-Tubulin served as the loading control. (e) Quantification of relative FoxO1 protein levels with densitometry. ImageJ $1.42 q$ software was used to analyze the density of each band in Figure $2 \mathbf{d}$, and the relative expression level was normalized to that of $\alpha$-tubulin. The data represent the mean \pm S.E. $(n=3)$. ${ }^{*} P<0.05$; ${ }^{* *} P<0.01$, Student's t-test

and caspase- 3 activity analysis showed that induction of the pro-apoptotic genes, $\mathrm{Bim}$ and caspase-3, by $\mathrm{H}_{2} \mathrm{O}_{2}$ was significantly decreased by FSH (Figures $3 e$ and f) in cultured MGCs. These observations were consistent with the results of in vivo experiments. To determine whether an indirect effect of FSH may influence MGC survival, we asked if FSH inhibited apoptosis by reducing oxidative stress. Our data showed no significant change in reactive oxygen species (ROS) levels following FSH treatment (Figures $3 g$ and $h$ ), suggesting a direct action of FSH on MGC protection. These results indicated that $\mathrm{FSH}$ decreased apoptosis in cultured MGCs.

FSH attenuated FoxO1 expression in cultured MGCs. Few reports have reported follicle stage-specific regulation of FoxO1 by $\mathrm{FSH}$ in granulosa cells. In this study, we determined whether FoxO1 expression was correlated with FSH in MGCs from DFs. FSH blocked the induction of FoxO1 in MGCs of DFs during the FSH withdrawal (Figures 2c-e). To validate the in vivo results, FoxO1 expression levels were examined in cultured MGCs isolated from ovarian DFs. As shown in Figure 4a, cells pre-treated with $\mathrm{H}_{2} \mathrm{O}_{2}$ displayed a marked decrease in FoxO1 mRNA (14.5-fold) following FSH incubation compared with the control group (1.6-fold), consistent with the observations from the TUNEL assay under the same conditions (Figures $3 c$ and $d$ ). Western blotting results also confirmed the inhibition of FoxO1 protein levels by FSH (Figures $4 \mathrm{~b}$ and $\mathrm{c}$ ). Taken together, the evidence from in vivo and in vitro experiments demonstrated that FSH repressed FoxO1-dependent apoptosis in MGCs of DFs.
FSH promoted MGC survival through the PKA-PI3KAKT-FoxO1 pathway. FSH activates multiple downstream signaling cascades in granulosa cells, including PKA, PI3K, $\mathrm{PKB} / \mathrm{AKT}$, p38-MAPK and ERK1/2. ${ }^{10}$ To further investigate the mechanisms of FoxO1 regulation by $\mathrm{FSH}$, we explored the role of these pathways in the inhibition of FoxO1 and apoptosis in primary cultured MGCs retrieved from DFs. qRTPCR analysis showed that H89 (PKA inhibitor) and LY294002 (PI3K inhibitor) abolished the downregulation of FoxO1 expression by FSH (Figures 5a and b), whereas SB203580 (p38-MAPK inhibitor) and U0126 (ERK1/2 inhibitor) had no effect (Figures $5 \mathrm{c}$ and d). Considering the role of PI3K/AKT in stimulating the cytoplasmic distribution and inactivation of FoxO $1,{ }^{15}$ we thus evaluated the impact of these pathways on FoxO1 subcellular localization. Immunofluorescence staining indicated that the actions of $\mathrm{FSH}$ in suppressing $\mathrm{H}_{2} \mathrm{O}_{2}-$ derived nuclear translocation of FoxO1 were reversed by $\mathrm{H} 89$ and LY294002, but not SB203580 or U0126 (Figures 5e and f). Consistent with this, compared with SB203580 and U0126, treatment with both $\mathrm{H} 89$ and LY294002 restored the $\mathrm{H}_{2} \mathrm{O}_{2}-$ triggered apoptosis, which was significantly decreased by FSH (Figure $5 \mathrm{~g}$ ). These data suggested a possible modulation by FSH of FoxO1-dependent apoptosis in MGCs through the PKA and PI3K/AKT pathways. We then examined the relationship among PKA, PI3K and AKT. As shown in Figures $5 \mathrm{~h}$ and $\mathrm{i}$, all three kinases were induced by FSH treatment. Blocking PKA or PI3K reduced AKT activity, but inactivation of PI3K and/or AKT had little effect on PKA activity. In contrast, the PKA inhibitor blunted the induction of both PI3K and AKT. Therefore, the PKA-PI3K-AKT-FoxO1 pathway may be involved in $\mathrm{FSH}$-mediated MGC protection. 
a

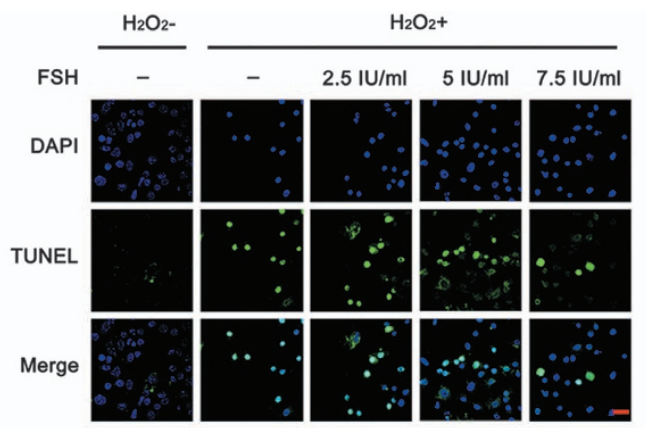

C
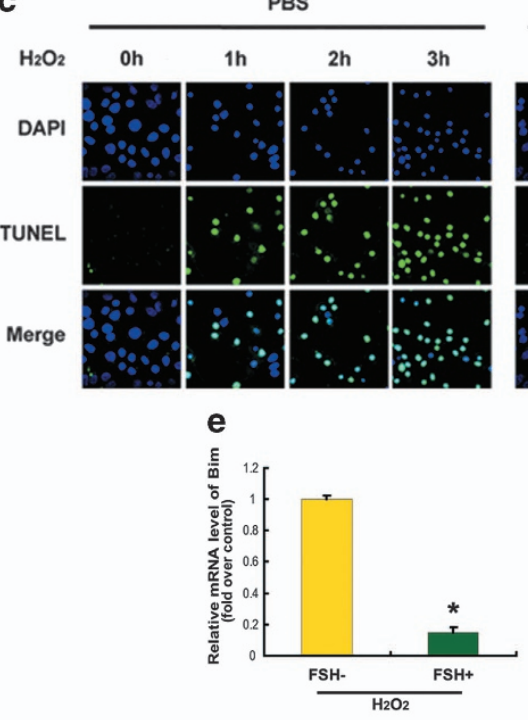

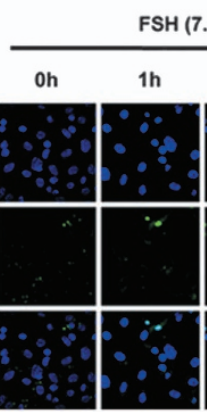

f

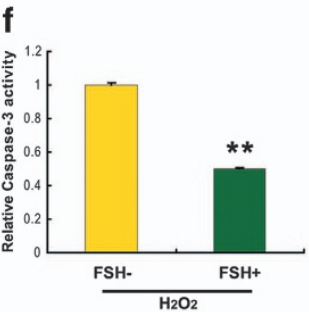

b

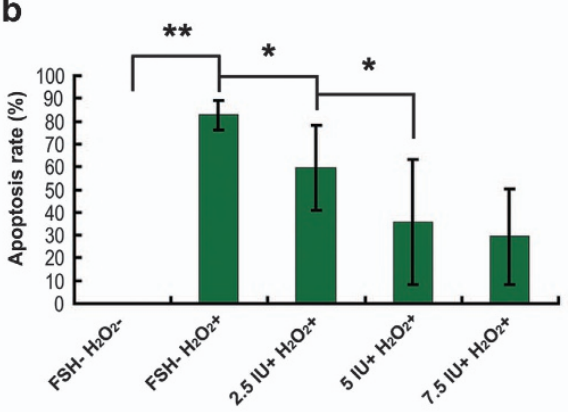

d

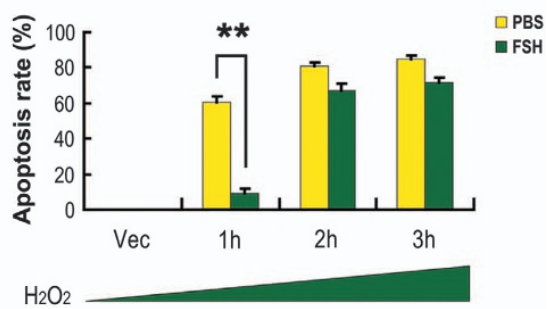

g $\mathrm{H}_{2} \mathrm{O}_{2}$

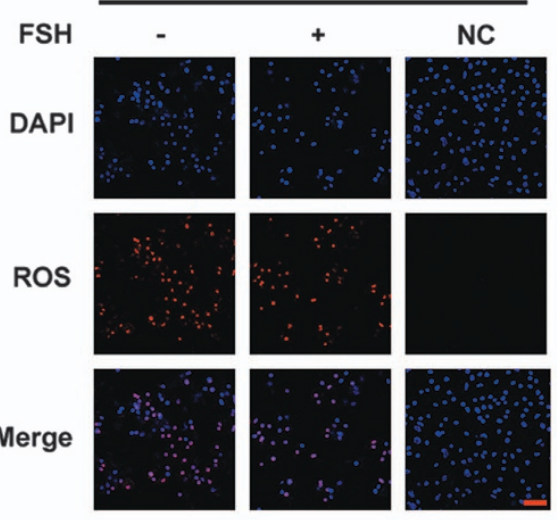

Figure 3 FSH suppressed apoptosis in cultured MGCs. (a) Granulosa cells collected from DFs were cultured in serum-free medium containing $200 \mu \mathrm{M} \mathrm{H} \mathrm{O}_{2}$ and FSH at different concentrations for $6 \mathrm{~h}$. The apoptotic signals were visualized with TUNEL staining (green), and the nuclei were counterstained with DAPI (blue). Bar, $20 \mu \mathrm{m}$. (b) Quantification of the apoptosis rates by calculating the average number of TUNEL-positive nuclei per visual field. Experiments were repeated three times, and cells were counted in three randomly selected fields from each coverslip. Data represent the means $\pm S$.E. The statistical significance between groups was analyzed with one-way ANOVA, and bars labeled with different letters differ significantly at $P<0.01$ (a and $\mathbf{b}$ ) or $P<0.05$ (a and $\mathbf{b}$ ). (c) After incubation with $200 \mu \mathrm{M} \mathrm{H}_{2} \mathrm{O}_{2}$ for 0 (vehicle), 1, 2 or $3 \mathrm{~h}, \mathrm{MGCs}$ were rinsed with PBS and cultured in serum-free medium containing $7.5 \mathrm{lU} / \mathrm{ml} \mathrm{FSH}$ for $12 \mathrm{~h}$. Apoptosis was detected as above. Bar, $20 \mu \mathrm{m}$. (d) Quantification of apoptosis rates in each treatment as shown in (c). Data represent the means \pm S.E. (e-g) MGCs were incubated for $1 \mathrm{~h}$ in $200 \mu \mathrm{M} \mathrm{H}_{2} \mathrm{O}_{2}$, washed with PBS, plated in serum-free medium with $7.5 \mathrm{IU} / \mathrm{ml}$ FSH for $12 \mathrm{~h}$, and then processed for qRT-PCR for Bim (e), caspase-3 activity assay (f) and intracellular ROS detection (g). Bar, $50 \mu \mathrm{m}$. (h) Quantification of intracellular ROS levels. The optical density was evaluated in each MGC with ImageJ $1.42 q$ software. Data represent mean \pm S.E. $(n=3)$. ${ }^{*} P<0.05$; ${ }^{* *} P<0.01$, Student's $t$-test

Constitutively active Fox01 in MGCs negated the protection from FSH. Our data revealed the FSH signaling that is required for downregulation of FoxO1 expression and apoptosis in MGCs of DFs. Our results in Figures $5 e$ and $f$ also suggested the influence of $\mathrm{FSH}$ on FoxO1 activity through PKA-PI3K-AKT. To further confirm the role that FoxO1 activity had in this pathway, we overexpressed wildtype (WT) FoxO1 (FoxO1-W) and a constitutively active FoxO1 mutant (FoxO1-AAA, the three AKT phosphorylation sites of FoxO1 are mutated to alanine) in cultured MGCs collected from DFs. Western blot analysis demonstrated a significant increase in FoxO1 expression after transfection. Equal amounts of FoxO1 protein levels were observed in both the FoxO1-W and FoxO1-AAA groups (Figures 6a and b), enabling us to specify the effects of FoxO1 activity without differences in FoxO1 expression. According to the TUNEL assay, both FoxO1 plasmids induced intense apoptotic signals in MGCs that were blocked by $\mathrm{FSH}$ in 


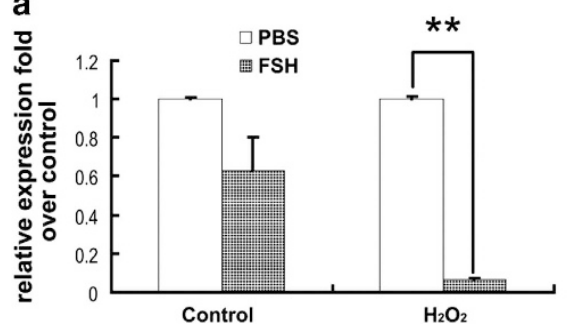

b

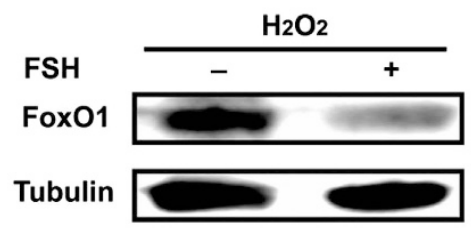

C

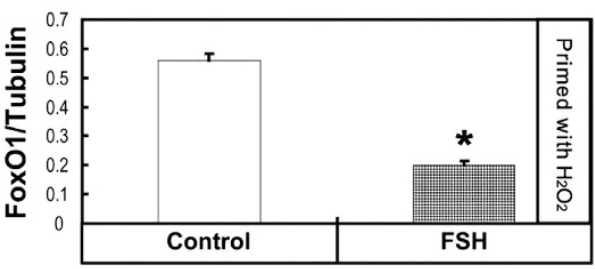

Figure 4 FoxO1 expression was downregulated by FSH in vitro. Primary MGCs collected from DFs were exposed to $200 \mu \mathrm{M} \mathrm{H}_{2} \mathrm{O}_{2}$ for $1 \mathrm{~h}$, rinsed with PBS and cultured in serum-free medium containing $7.5 \mathrm{IU} / \mathrm{ml} \mathrm{FSH}$ for $12 \mathrm{~h}$. (a) The effects of FSH on FoxO1 expression in MGCs were determined with qRT-PCR. Expression data were normalized to that of $\beta$-actin. (b) Western blotting analysis of FoxO1 protein levels in cultured MGCs treated with or without FSH. $\alpha$-Tubulin was used as the loading control. (c) Quantification of the relative FoxO1 protein level with densitometry. ImageJ 1.42q software was used to analyze the density of each band in Figure $4 \mathbf{b}$, and the relative expression level was normalized to that of $\alpha$-tubulin. The data represent the mean \pm S.E. $(n=3)$. ${ }^{*} P<0.05$; ${ }^{\star \star} P<0.01$, Student's $t$-test

the FoxO1-W group. Conversely, expression of FoxO1-AAA abolished FSH-induced cell survival (Figures $6 \mathrm{c}$ and $\mathrm{d}$ ). In accordance with this, immunofluorescence staining indicated that FSH inhibited overexpression-triggered nuclear translocation of FoxO1 in the FoxO1-W group, but not in the FoxO1-AAA group (Figures $6 e$ and $\mathrm{f}$ ). These observations suggested the importance of FoxO1 activity in the response to the FSH-activated PKA-PI3K-AKT axis in MGCs.

The self-reinforcing regulation of FoxO1 in MGCs. As both FoxO1 expression and activity participated in $\mathrm{FSH}$ initiated apoptotic control in MGCs through the PKA-PI3KAKT axis, we next sought to examine their connection in this process. FoxO3a was previously reported to bind to the FoxO1 promoter region and control FoxO1 expression. ${ }^{36-38}$ As all FoxO family members have a consensus DNA-binding sequence known as $\mathrm{FRE},{ }^{15}$ we hypothesized that FoxO1 triggered apoptosis by upregulating FoxO1 expression in MGCs of DFs when FSH signaling was absent. To test this hypothesis, MGCs retrieved from DFs were transfected with pcDNA3-FLAG-FKHR (W) or pcDNA3-FLAG-FKHR;H215R ( $F$, containing a point mutation in the DNA-binding domain (DBD)). As shown in the western blot, overexpression of WT FoxO1 significantly increased endogenous FoxO1 expression, whereas the DBD mutant had no effect (Figures $7 a$ and $b$ ). In accordance with this, the DBD mutant lost the activator effect of FoxO1 overexpression on apoptosis in MGCs (Figures 7c and d). In addition, considering that FRE sites are also found in the Bim promoter, ${ }^{18}$ we measured Bim expression with qRT-PCR under the same conditions. Compared with MGCs transfected with WT FoxO1, DBD mutation had little effect on the induction of intracellular mRNA levels of Bim (Figure 7e). Collectively, our data indicated that FoxO1 enhanced the expression of FoxO1 and its downstream apoptotic gene, thus leading to apoptosis in MGCs.

To further test if FoxO1 directly activated the expression of its own gene at the promoter level, a 2048-bp fragment of the FoxO1 5' UTR containing the putative FRE site was cloned in front of a luciferase reporter gene as pGL3-FoxO1 (W) (Figure $8 \mathrm{a}$ and Supplementary Figure S3). This construct was then co-transfected with pRL-TK and pcDNA3-FLAGFKHR (W) or the control pcDNA3 into NIH 3T3 fibroblasts. WT FoxO1 but not the pcDNA3 vector markedly heightened
FoxO1 promoter activity. In contrast, FRE site mutation significantly decreased the responsiveness of the reporter to FoxO1 overexpression (Figure 8b). We next performed chromatin immunoprecipitation (ChIP) experiments to investigate the binding of FoxO1 to the FoxO1 promoter. MGC and $\mathrm{NIH} 3 \mathrm{~T} 3$ fibroblast chromatin was first sheared into fragments of 250-750 bp with sonication (Supplementary Figure S6) and then used for immunoprecipitation. As shown in Figures 8c-e, the anti-FoxO1 antibody precipitated the FoxO1 promoter region containing the FRE site in chromatin from both MGCs and NIH 3T3 fibroblasts. Importantly, the signals in MGCs were markedly inhibited following $\mathrm{FSH}$ treatment for $6 \mathrm{~h}$. Together, these results suggested that FoxO1 was directly recruited to the FRE site in the FoxO1 promoter for transcriptional activation in the absence of $\mathrm{FSH}$.

Moreover, we asked if FSH signaling affected FoxO1 expression via other transcriptional factors. We first predicted the transcriptional factor binding sites in the FoxO1 promoter using TFSEARCH, Promoter Scan and GeneCards and selected candidate genes for qRT-PCR analysis. Of the six genes with detectable mRNA levels in MGCs, a strong correlation between the expression patterns of estradiol receptor $(\mathrm{ER})$ alpha and FoxO1 $(P<0.01)$ in response to FSH and PKA inhibitor treatment was observed (Supplementary Figure S4), indicating that ERalpha may also be required for FoxO1 induction upon FSH deprivation.

In conclusion, our data demonstrated that the PKA-PI3KAKT-FoxO1 axis and FoxO1-FoxO1 positive feedback were important mechanisms for FSH-mediated MGC survival in ovarian DFs.

\section{Discussion}

The regulation of follicular atresia requires complex interactions between gonadotropins (FSH and $\mathrm{LH}$ ) and ovarian regulatory factors ${ }^{39}$ for determination of granulosa cell fate (proliferation, differentiation or programmed cell death). ${ }^{6}$ Interestingly, as a transcription factor responsible for cell growth arrest and apoptosis in multiple types of mammalian cells, ${ }^{40}$ FoxO1 is selectively and highly expressed in granulosa cells of mammalian growing follicles. ${ }^{39}$ In MGCs, FoxO1 activity is controlled by steroid hormones, ${ }^{39}$ whereas overexpression of FoxO1 reduces steroidogenesis. ${ }^{22}$ In addition, immunohistochemistry showed intense nuclear staining of 
a

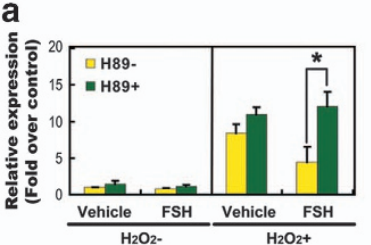

b

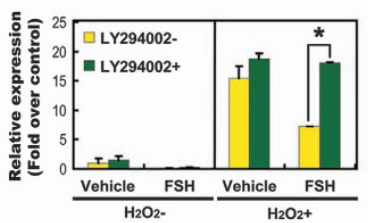

C

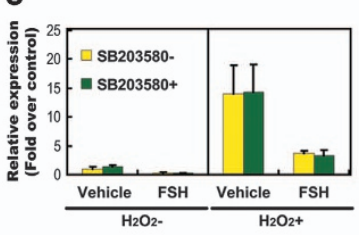

d

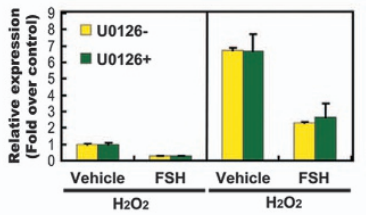

e

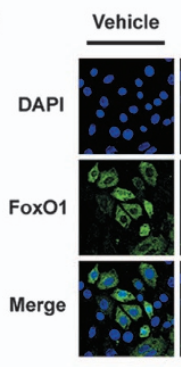

FSH

$\mathrm{H}_{2} \mathrm{O}_{2}$

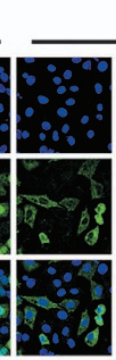

$\mathrm{H}_{2} \mathrm{O}_{2}+\mathrm{FSH}$

f
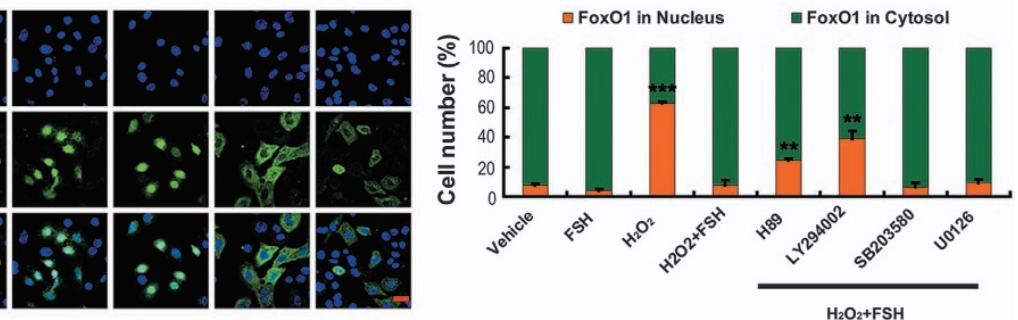

$\begin{array}{ccc}\text { H89 } & - & - \\ \text { LY294002 } & - & - \\ \text { SB203580 } & - & - \\ \text { U0126 } & - & -\end{array}$

g

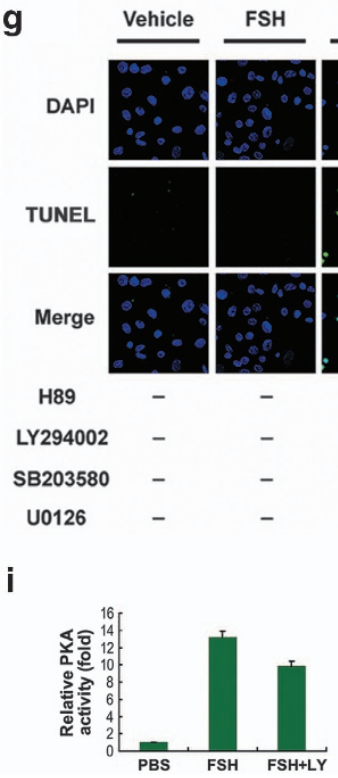

FSH

$\mathrm{H}_{2} \mathrm{O}_{2}$

$\mathrm{H}_{2} \mathrm{O}_{2}+\mathrm{FSH}$
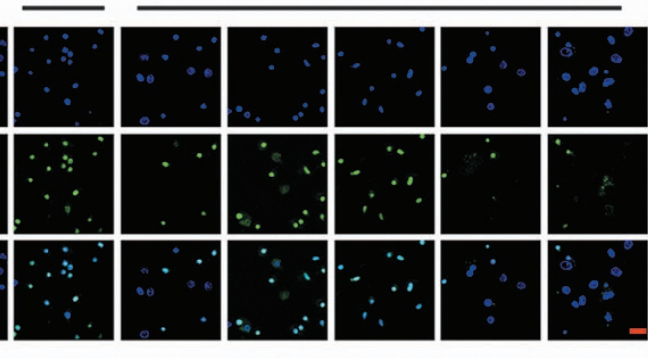

h
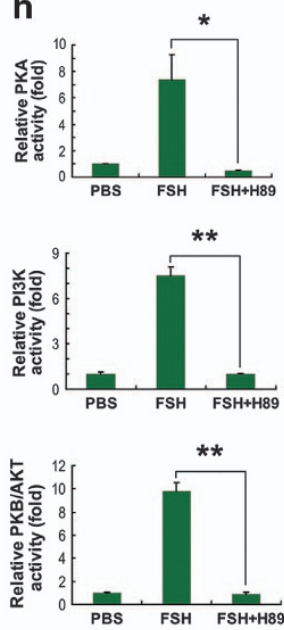

$-$

$-$

$+$

$-$

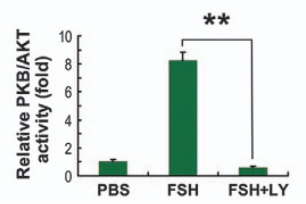

Figure 5 FSH prevented MGCs from undergoing apoptosis via the PKA-PI3K-AKT-FoxO1 axis. Primary cultured MGCs were exposed to $200 \mu \mathrm{M} \mathrm{H} \mathrm{H}_{2}$ for $1 \mathrm{~h}$ and then grown in serum-free medium containing $7.5 \mathrm{IU} / \mathrm{ml} \mathrm{FSH}$ for $2 \mathrm{~h}$ (in $\mathbf{e}, \mathbf{f}, \mathbf{h}$ and i) or $6 \mathrm{~h}$ (in a-d and g). H89 (10 $\mu \mathrm{M})$, LY294002 $(20 \mu \mathrm{M}), \mathrm{SB} 203580(20 \mu \mathrm{M})$ or U0126 $(3 \mu \mathrm{M})$ was added for 30 min before FSH treatment. (a-d) The effects of the FSH signaling antagonists on FoxO1 expression were determined with qRT-PCR. Expression data were normalized to that of $\beta$-actin. (e) Subcellular localization of FoxO1 in response to the indicated FSH signaling inhibitors was detected using anti-FoxO1 (green), and the nuclei were counterstained with DAPI (blue). Bar, $20 \mu \mathrm{m}$. (f) The percentage of cells with FoxO1 in the nucleus (orange bars) and in the cytosol (green bars). Experiments were performed in triplicate, and cells were counted in three randomly selected fields in each coverslip. Data represent the mean \pm S.E. (g) The effects of the FSH signaling antagonists on stress-induced apoptosis in cultured MGCs. The apoptotic signals were visualized with TUNEL staining (green), and the nuclei were counterstained with DAPI (blue). Bar, $20 \mu \mathrm{m}$. (h) The effects of the PKA inhibitor H89 on the activity of PKA, PI3K and AKT. (i) The effects of the PI3K inhibitor LY294002 on the activity of PKA, PI3K and AKT. The data represent the mean \pm S.E. $(n=3) .{ }^{*} P<0.05 ;{ }^{* \star} P<0.01 ;{ }^{* \star \star} P<0.001$, Student's $t$-test

FoxO1 that was centralized in rat granulosa cells from atretic follicles. ${ }^{23}$ Our previous work also identified FoxO1 as a critical factor in oxidative stress-triggered MGC apoptosis. ${ }^{4}$ These reports suggested a possible contribution of FoxO1 to follicular atresia. $^{22,25}$ Nevertheless, no direct evidence has been obtained indicating that FoxO1 performs the same role under physiological conditions. Gonadotrophins (FSH and LH) are primarily responsible for the selection of DFs. ${ }^{41} \mathrm{FSH}$ alone promotes antral follicle growth and development into preovulatory follicles, which will maintain anovulation without stimulation by $\mathrm{LH}^{29} \mathrm{FSH}$ withdrawal (coasting) during this stage leads to granulosa cell apoptosis and follicular atresia. ${ }^{30}$ 


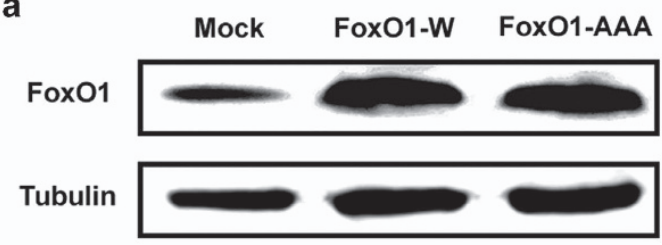

C

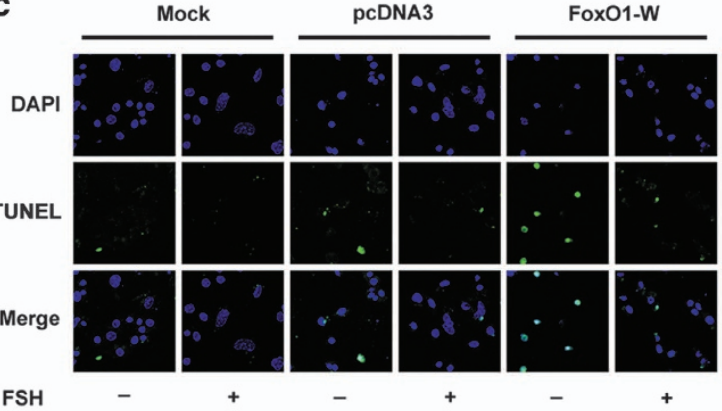

Fox01-AAA

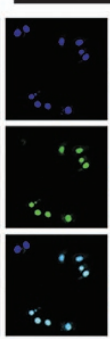

$-$ b

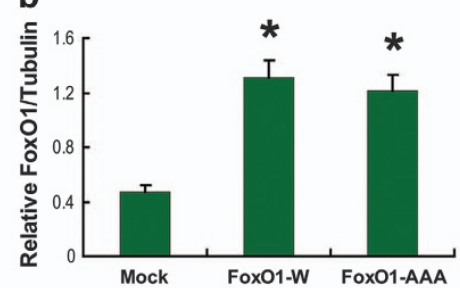

d

$\square$ FSH-

- FSH+

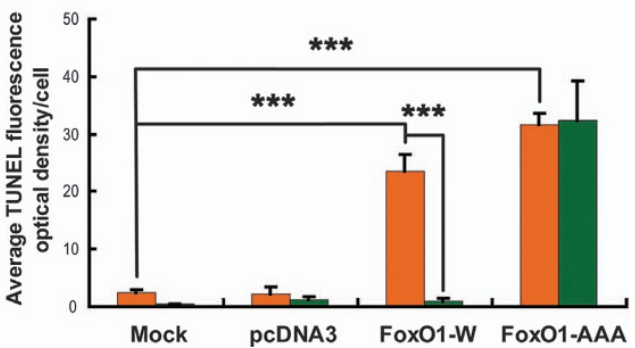

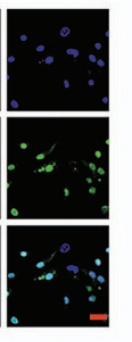

e
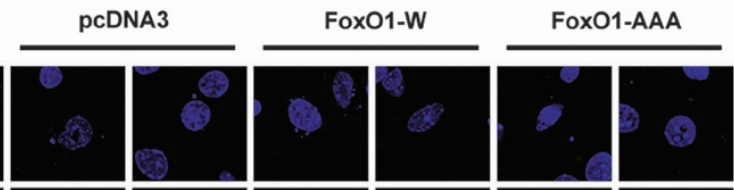

Fox01
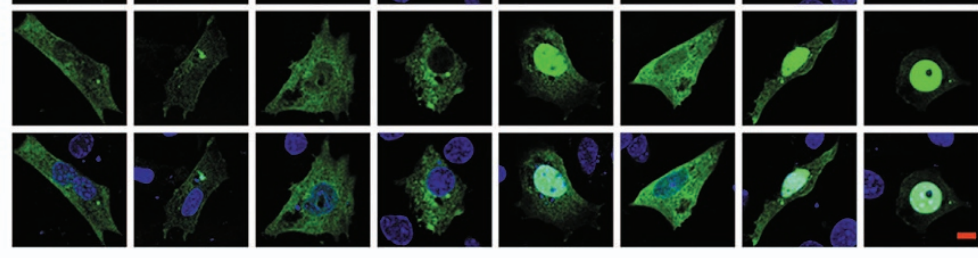

FSH

f

$\square$ FoxO1 in Nucleus FSH- $\quad$ FoxO1 in Cytosol FSH-

$\square \mathrm{FoxO} 1$ in Nucleus FSH+ $\square \mathrm{FoxO} 1$ in Cytosol FSH+

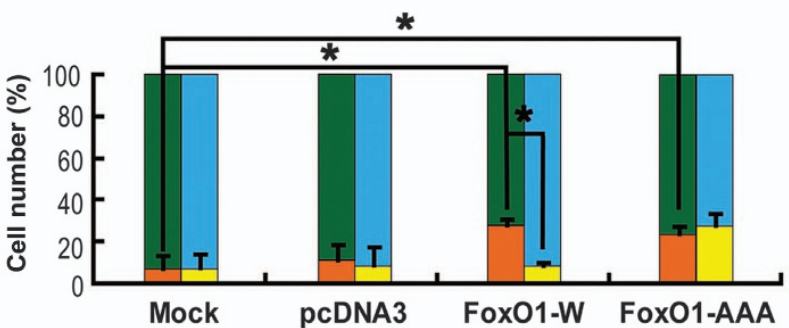

Figure 6 Activation of FoxO1 in MGCs abolished the anti-apoptotic effects of FSH. Primary MGCs were transfected with WT FoxO1 (FoxO1-W), a constitutively active FoxO1 mutant (FoxO1-AAA, all three AKT sites were replaced by alanines) or a control vector (pcDNA3) for $36 \mathrm{~h}$. (a) Western blotting analysis of FoxO1 protein levels in cultured MGCs transfected with FoxO1 expression vectors. $\alpha$-Tubulin was used as the loading control. (b) Quantification of relative FoxO1 protein levels with densitometry. ImageJ 1.42q software was used to analyze the density of each band, and the relative expression level was normalized to that of $\alpha$-tubulin. (c) Cells were treated with or without FSH (7.5 IU/ml) during transfection. The apoptotic signals were detected with FITC fluorescence (green), and the nuclei were counterstained with DAPI (blue). Bar, $20 \mu \mathrm{m}$. (d) Quantification of the apoptotic signals. The optical density was evaluated in each MGC with ImageJ 1.42q software. (e) Cells were incubated with or without FSH (7.5 IU/ml) before collection. Subcellular localization of FoxO1 upon FSH stimulation was visualized using anti-FoxO1 (green), and the nuclei were counterstained with DAPI (blue). Bar, $10 \mu \mathrm{m}$. (f) The percentage of cells with FoxO1 in the nucleus or the cytosol under the indicated conditions. Experiments were performed in triplicate, and cells were counted in three randomly selected fields on each coverslip. ${ }^{*} P<0.05 ;{ }^{* * *} P<0.001$, Student's $t$-test

We therefore developed a corresponding FSH treatment protocol to mimic in vivo growth and atresia of DFs (Materials and Methods section and Supplementary Figure S1). According to our treatment procedure, we observed a clear association between MGC apoptosis and FoxO1 expression in DFs (Figures 1 and 2), providing new evidence for the proapoptotic effect of FoxO1 in MGCs.

Previous studies indicated that the actions of $\mathrm{FSH}$ on ovarian functions are partially mediated by FoxO1. ${ }^{23,39}$ For example, the $\mathrm{FSH}$-induced expression of genes required for 
a

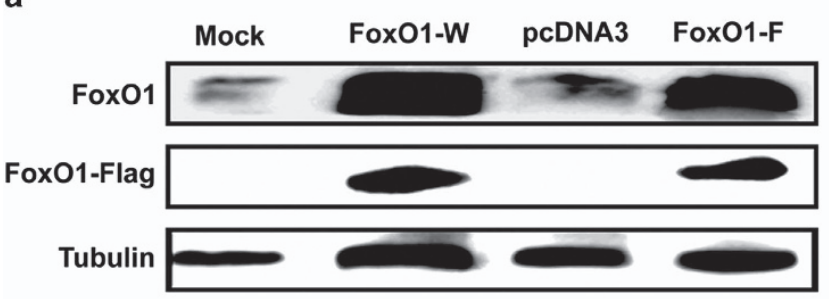

b

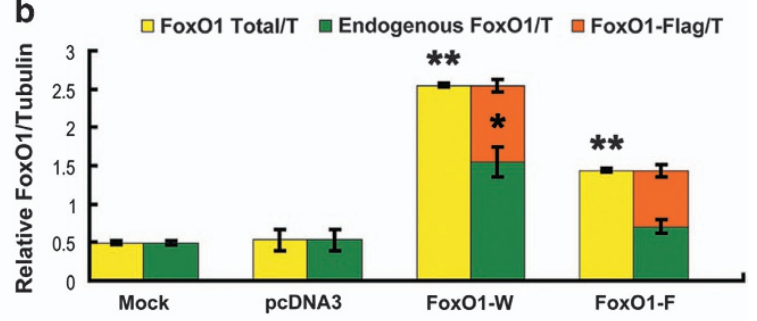

C
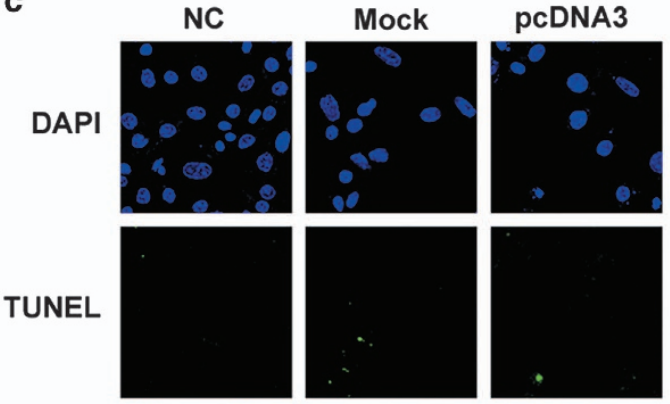

FoxO1-W

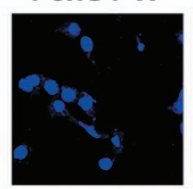

Fox01-F
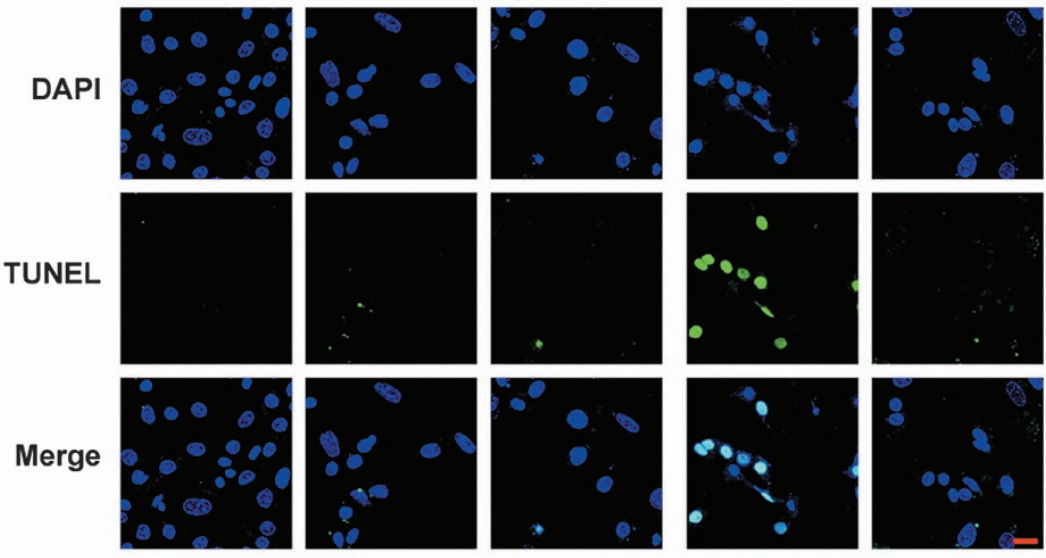

d

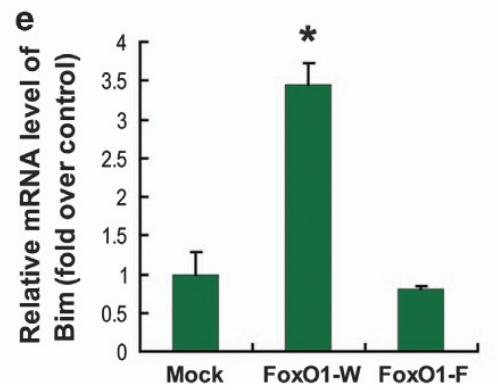

Figure 7 Fox01-induced apoptosis in MGCs by activating the expression of FoxO1 and downstream apoptotic genes. Primary cultured MGCs harvested from DFs were transfected with pcDNA3-FLAG-FKHR (FoxO1-W), pcDNA3-FLAG-FKHR;H215R (FoxO1-F, containing a point mutation in the DBD), or a control vector (pcDNA3) for 36 $\mathrm{h}$. (a) Western blotting analysis of FoxO1 protein levels. $\alpha$-Tubulin was used as the loading control. (b) Quantification of relative FoxO1 protein levels with densitometry. ImageJ $1.42 q$ software was used to analyze the density of each band, and the relative expression level was normalized to that of $\alpha$-tubulin. Yellow bar, total FoxO1 protein. Green bar, endogenous FoxO1 protein. Orange bar, exogenous FoxO1 protein. (c) The apoptotic signals in MGCs after transfection were detected with FITC fluorescence (green), and the nuclei were counterstained with DAPI (blue). Bar, $20 \mu \mathrm{m}$. (d) Quantification of the apoptotic signals. The optical density was evaluated in each MGC with ImageJ 1.42q software. Experiments were performed in triplicate, and cells were counted in three randomly selected fields in each coverslip. Data represent the means $\pm S$.E. (e) Transcription changes in Bim in response to FoxO1 overexpression-induced apoptosis in MGCs. Expression data were normalized to that of $\beta$-actin. ${ }^{*} P<0.05$; ${ }^{*} P<0.01$, Student's $t$-test

lipid, sterol and steroid synthesis in MGCs is attenuated by a constitutively active FoxO1 mutant. ${ }^{22}$ In contrast, FSH blocks the inhibition of granulosa cell proliferation and estrogen production from FoxO1. 22,24 Further research in granulosa cells showed that FoxO1 represses transcription of cyclin D2, thereby blocking cell cycle progression at the G0 phase. Also, FoxO1 prevents granulosa cell differentiation by downregulating steroidogenic factor-1, inhibin- $a$, aromatase cytochrome P-450 and epiregulin. ${ }^{25}$ Therefore, FSH-mediated FoxO1 suppression will promote granulosa cell proliferation and differentiation. ${ }^{10}$ However, whether FoxO1 is correlated with the effects of FSH on antagonizing granulosa cell apoptosis remains to be determined. In this study, we demonstrated that FSH significantly decreased granulosa cell apoptosis and/or follicular atresia in the mouse ovary (Figures 1 and 3), consistent with previous reports. ${ }^{32,42-44}$ Importantly, MGC apoptosis that was reduced by $\mathrm{FSH}$ treatment was associated with downregulation of FoxO1 expression (Figures 2 and 4). Thus, FSH may impair apoptosis in MGCs in a FoxO1dependent manner.

Mammalian ovaries contain follicles in various stages of development. ${ }^{9}$ Although FoxO1 is a target of $\mathrm{FSH}$ signaling, ${ }^{25,39,45}$ few studies have been performed on the follicle stage-specific modulation of FoxO1 by FSH. Studies from others demonstrated that FSH treatment significantly decreases FoxO1 levels in cultured granulosa cells. However, exposure of developing follicles to FSH enhances the expression of FoxO1, which is downregulated as granulosa cells differentiate to luteal cells. ${ }^{22,24,26,27,39}$ These contradictory observations suggested that $\mathrm{FSH}$ has distinct effects 
a

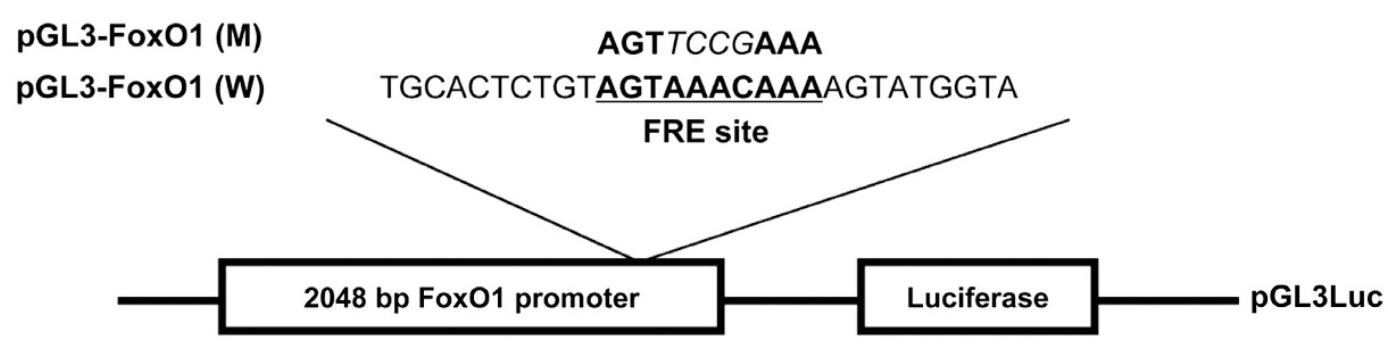

b $\square$ pcDNA3 圆 pcDNA3-FLAG-FKHR (WT)

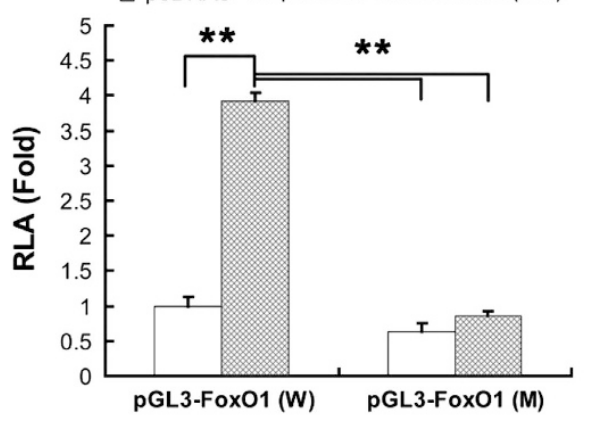

C

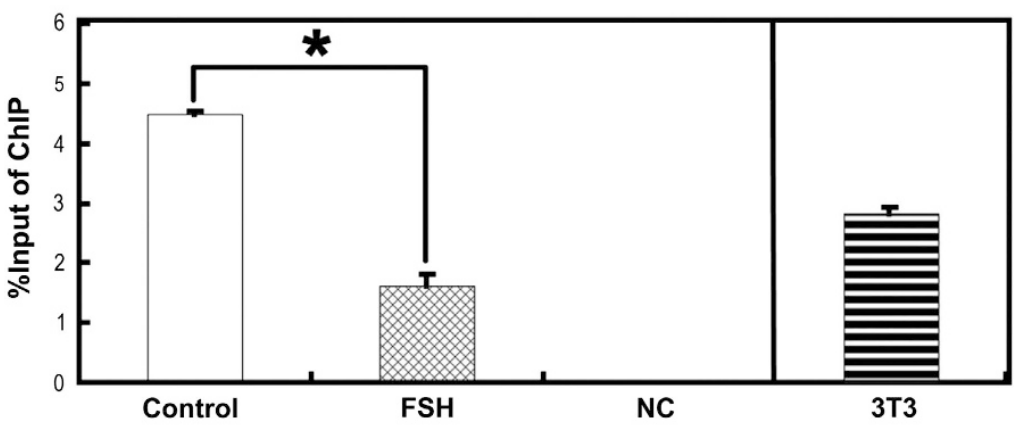

d
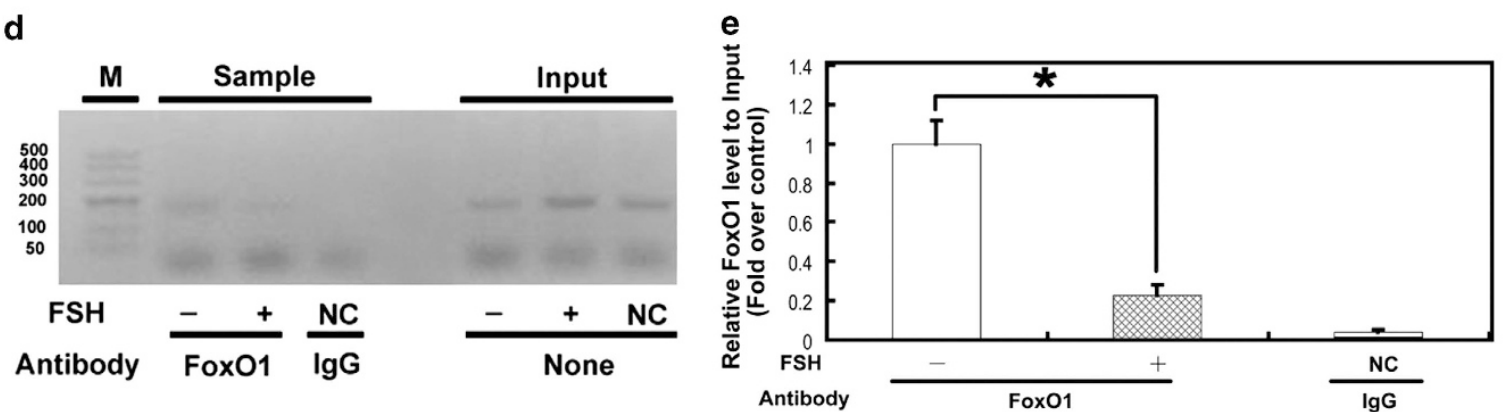

Figure 8 FoxO1 directly bound to the FRE site in the FoxO1 promoter. (a) A 2048-bp fragment of the FoxO1 promoter was amplified with PCR using mouse genomic DNA (bases 78-2125, 5'-UTR of Fox01) containing a FRE (AGTAAACAAA) and cloned into the pGL3-Basic plasmid as pGL3-Fox01 (W). pGL3-FoxO1 (M) was obtained by introducing four mutations (italics) into the FRE site. (b) FoxO1 reporter activities in mouse $3 \mathrm{~T} 3$ cells co-transfected with FoxO1 expression vectors and FoxO1 promoter constructs for $36 \mathrm{~h}$. The reporter activities were normalized to those of pRL-TK. RLA, relative luciferase activities. (c-e) The binding of Fox01 to the Fox01 promoter in MGCs or 3T3 fibroblasts was detected with ChIP assays following FSH treatment for $6 \mathrm{~h}$. DNA was isolated from the precipitated complexes as a template for qRT-PCR (c). The qRT-PCR products were then analyzed on a $2 \%$ agarose gel (d) and quantified with densitometry using ImageJ 1.42q software (e). The amount of immunoprecipitated DNA for each ChIP reaction is shown as the percentage of input chromatin. IgG was the negative control. The data represent the means $\pm S . E$. $(n=3)$. ${ }^{*} P<0.05$; ${ }^{* *} P<0.01$, Student's $t$-test

on FoxO1 expression in granulosa cells at different follicular stages, and thus, we explored the correlation between FoxO1 and FSH in MGCs from DFs. Our data indicated a synchronization of protection of MGCs and/or DFs and FoxO1 inhibition that was mediated by FSH (Figures 1-4), supporting the concept that FSH is the major survival factor for DFs. ${ }^{28}$

Two major cascades lead to apoptosis: the extrinsic pathway (type I cell death pathway), which activates cell surface receptors in response to external signals such as FasL, and the intrinsic pathway (type II cell death pathway), which causes cytochrome $\mathrm{c}$ release into the cytosol following mitochondrial membrane disruption that is induced by $\mathrm{Bcl}-2$ family members. ${ }^{46}$ Previous studies identified Bim as a $\mathrm{BH} 3-$ only protein that promotes apoptosis by changing the balance between pro- and anti-apoptotic members of the Bcl-2 family, thereby affecting permeability of the mitochondrial membrane to cytochrome $\mathrm{c}$, which further triggers the activation of caspase-9, caspase-3 and the cell death program. ${ }^{47}$ Initiation of apoptosis occurs in granulosa cells through the mitochondria-dependent pathway. ${ }^{31}$ As shown in this study (Figure 1) together with our previous results, ${ }^{32}$ MGC apoptosis driven by FSH withdrawal induced hallmarks of mitochondrial apoptosis (Bim, caspase-9 and caspase-3), which were then suppressed in the presence of FSH. In contrast, FSH had no effects on the components of death receptor signaling (FasL and caspase-8). These data suggested the involvement of the type II cell death pathway in FSH-mediated MGC protection. In addition, previous evidence indicated that FoxO1 regulates apoptosis by targeting several pro-apoptotic genes, including Bim. ${ }^{18,19,48-50}$ Our results showed that Bim followed a similar expression pattern as FoxO1 during $\mathrm{FSH}$ treatment. Therefore, Bim appears to be a convergence point of the 
mitochondrial apoptotic pathway and FoxO1-dependent apoptosis that both contribute to FSH-induced MGC survival in DFs.

$\mathrm{FSH}$ regulates the growth and differentiation of follicular granulosa cells through several downstream signaling pathways, including PKA, PI3K, AKT, p38-MAPK and ERK1/2, although their interactions in response to $\mathrm{FSH}$ remain to be investigated. ${ }^{10}$ Previous studies indicated that FoxO1, the major downstream effector of $\mathrm{PI} / \mathrm{K} / \mathrm{AKT}$, is phosphorylated following exposure to FSH in granulosa cells. ${ }^{25,39,45}$ In this study, it was found that both PKA and PI3K/AKT were activated by $\mathrm{FSH}$ in MGCs of DFs to inhibit the expression of FoxO1 following stress-triggered apoptosis (Figures $5 \mathrm{a}$ and b). In contrast, H89 (PKA inhibitor) and LY294002 (PI3K inhibitor) diminished the protection against apoptosis provided by FSH in cultured MGCs that was associated with suppression of FoxO1 activity (Figures 5e-g). Correspondingly, overexpression of a constitutively active FoxO1 mutant (all three AKT sites were replaced by alanines) in MGCs abolished the actions of FSH on repressing FoxO1 and apoptosis (Figure 6). Further experiments using FSH signaling antagonists identified PKA as an upstream activator of PI3K, which in turn stimulated the activation of AKT (Figures $5 \mathrm{~h}$ and i). These findings were consistent with a role for PKA in initializing $\mathrm{FSH}$ induced granulosa cell survival as described previously. ${ }^{51-53}$ MAPK-related proteins, such as JNK, ERK1/2 and p38MAPK, regulate FoxO1 activity, ${ }^{54}$ stress resistance ${ }^{55}$ and $\mathrm{FSH}$ response. ${ }^{56}$ However, no definitive evidence was observed for the involvement of either ERK1/2 or p38-MAPK in preventing MGC apoptosis or FoxO1 induction with $\mathrm{FSH}$ treatment (Figures 5c-g). Taken together, our results suggested that PKA-PI3K-AKT-FoxO1 may be a major axis for FSHmediated MGC protection.

The potent functions of FoxO1 are tightly controlled by its transcription and phosphorylation states. ${ }^{40,57}$ Although it is well understood that phosphorylation of FoxO1 by PKB/AKT protects cells from apoptosis because of the nuclear exclusion of phospho-FoxO1 and thereby blocking transactivation of proapoptotic genes including p27KIP1, Bim, TRAIL and FasL, ${ }^{16-20}$ the interplay between phosphorylation and expression in the regulation of FoxO1 expression has not been entirely characterized, especially in granulosa cells. ${ }^{39}$ As mentioned above in this study, the inactivation of FoxO1 by PKA-PI3KAKT may also be attributed to its phosphorylation and subsequent sequestration in the cytoplasm (Figures 5 and 6). However, mechanisms regarding the inhibition of FoxO1 expression through this signaling axis remain to be explored. Our results showed that overexpression of WT FoxO1 stimulated endogenous FoxO1 expression and FoxO1 promoter activity, as well as elevation of apoptotic signals in MGCs (Figures 7 and 8). In fact, FoxO1 bound directly to the FRE site within the FoxO1 promoter (Figure 8), whereas phosphorylation of FoxO1 by FSH dissociated FoxO1 from its DNA-binding sequence, which was correlated with the downregulation of FoxO1 expression (Figures 2,4-6 and 8). Therefore, FSH-induced FoxO1 suppression may also terminate the transcriptional induction of its downstream proapoptotic effectors such as Bim (Figures 3 and 7). These data suggested that when FSH signaling is absent, FoxO1 activates the transcription of FoxO1 and its downstream apoptotic genes, thereby triggering apoptosis in MGCs. Consistent with our observations, earlier reports indicated the ability of FoxO3a to bind and activate both the FoxO1 and FoxO3a promoters in other cell types. ${ }^{36-38,58}$ As all FoxO family members share the FRE consensus DNA-binding sequence, ${ }^{15}$ such positive feedback regulation may be a common mechanism among the FoxO genes. A recent study in porcine granulosa cells showed that FSH inhibits nuclear accumulation of FoxO3a by PI3K/AKT-mediated phosphorylation. ${ }^{13}$ Therefore, although FSH has little effect on the expression of FoxO3a (Figure 2b), whether FSHregulated FoxO3a activity exerts any influence on FoxO1 expression remains to be investigated in MGCs. Nevertheless, our study sheds new light on the connection between FoxO1 expression and FoxO1 activity in $\mathrm{FSH}$-mediated MGC survival. A hypothetical model (Supplementary Figure S5) shows $\mathrm{FSH}$ regulation of FoxO1-dependent apoptosis in MGCs of ovarian DFs.

Moreover, FSH stimulates granulosa cells to secrete estradiol, a steroid hormone that promotes ovarian follicle survival and growth via two ERs, ERalpha and ERbeta. ${ }^{59}$ In this study, a positive correlation between FoxO1 and ERalpha expression was seen in response to FSH and PKA inhibitor treatment both in vitro (Supplementary Figure S4) and in vivo (data not shown) in MGCs. Previous reports indicated that ERalpha and FoxO1 proteins are downstream targets of estradiol. ${ }^{60}$ Proteomic analysis showed the binding of ERalpha and FoxO1 on estrogen response elements. ${ }^{61}$ Similarly, FoxO1 colocalizes with ERalpha in the nucleus of breast cancer cells, but estradiol-induced FoxO1 phosphorylation led to nuclear export of the FoxO1-ERalpha complex. ${ }^{62}$ Although these findings demonstrated coregulation of ERalpha and FoxO1, our results suggest a relationship at the transcription level. However, further experiments such as ChIP, luciferase reporter assays, qRT-PCR, western blotting and TUNEL staining should be conducted to confirm the effects of ERalpha on FoxO1 expression during FSH-induced MGC survival.

In summary, this study describes a mechanism underlying FSH-mediated protection against apoptosis in MGCs by suppressing FoxO1 through the PKA-PI3K-AKT-FoxO1 axis and FoxO1-FoxO1 positive feedback. Further identifying the functions of the upstream regulators and downstream effectors of FoxO1 should provide novel insights into the exact regulation modes of FoxO1-dependent apoptosis upon FSH stimulation during follicular atresia. Therefore, targeting FSH-FoxO1 signaling may be beneficial in clinical therapy for follicular atresia-related disorders such as polycystic ovarian syndrome and premature ovarian failure.

\section{Materials and Methods}

Animals. All the animal experiments were performed in accordance with the guidelines of the Animal Research Institute Committee at Nanjing Agricultural University. Three-to-four-week-old female ICR mice (Qing Long Shan Co., Animal Breeding Center, Nanjing, China) were housed five per cage in a temperaturecontrolled $\left(22 \pm 2{ }^{\circ} \mathrm{C}\right)$ room with a $12: 12 \mathrm{~h}$ light:dark cycle (lights on from 0700 to 1900 hours) and had ad libitum access to water and food. FSH administration was performed as previously described (also see Supplementary Figure S1). ${ }^{32}$ Briefly, to induce growth of DFs, mice were i.p. injected with FSH (Ningbo Second Hormone Factory, Ningbo, China) twice daily (12-h intervals, 0800 and 2000 hours) for 2 days at a dose of $10 \mathrm{IU}$ on day 1 and $5 \mathrm{IU}$ on day 2. FSH was then withdrawn for an additional 24 or $48 \mathrm{~h}$ to promote natural follicular atresia in DFs, or injected i.p. 
(10 IU per mouse) $6 \mathrm{~h}$ before MGC retrieval. At 48,72 and $96 \mathrm{~h}$ after the first FSH injection, MGCs were isolated from DFs $(>200 \mu \mathrm{m})$ in the left ovaries for qRT-PCR, immunoblotting and caspase activity detection. The right ovaries were fixed with $4 \%$ paraformaldehyde and embedded in paraffin for subsequent TUNEL assay and H\&E staining. In this study, unless otherwise specified, MGCs were equal to MGCs from DFs.

Cell culture. For primary MGC culture, the procedures were performed as described. ${ }^{4}$ In brief, 3- to 4-week-old ICR mice were injected i.p. with $10 \mathrm{IU}$ pregnant mare serum gonadotropin and killed $48 \mathrm{~h}$ later. ${ }^{63}$ Superovulated mouse ovaries were harvested and individually transferred into $35-\mathrm{mm}$ Petri dishes containing PBS and then punctured with a syringe to release MGCs from DFs ( $>200 \mu \mathrm{m}$ in diameter) under a surgical dissecting microscope. The cell suspensions were plated in DMEM/F-12 (1:1) (Invitrogen, Shanghai, China) supplemented with 10\% fetal bovine serum (FBS; Gibco, Grand Island, NY, USA) and 100 units/ml penicillin plus $100 \mu \mathrm{g} / \mathrm{ml}$ streptomycin (Gibco). After incubation for 4 days at $37^{\circ} \mathrm{C}$ in $5 \% \mathrm{CO}_{2}$, MGCs were washed and cultured in the presence or absence of FSH (ProSpecbio, Ness-Ziona, Israel) for the indicated times. NIH 3T3 cells (mouse embryonic fibroblasts, Institute of Biochemistry and Cell Biology, CAS, Shanghai, China) were grown in DMEM high-glucose medium (Invitrogen) with 10\% FBS (Gibco) following previously reported protocols. ${ }^{64}$

\section{Cell treatments and transfection}

FSH treatment: H89, LY294002, SB203580 and U0126 were purchased from Beyotime (Beijing, China). After exposure to $200 \mu \mathrm{M} \mathrm{H}_{2} \mathrm{O}_{2}$ (Sigma, St. Louis, MO, USA) for $1 \mathrm{~h}, \mathrm{MGCs}$ were rinsed with PBS and grown in serum-free DMEM/F-12 containing $7.5 \mathrm{IU} / \mathrm{ml} \mathrm{FSH}$ for 2, 6, 12, 24 or $36 \mathrm{~h}$. In some experiments, H89 $(10 \mu \mathrm{M})$, LY294002 $(20 \mu \mathrm{M})$, SB203580 $(20 \mu \mathrm{M})$ or U0126 $(3 \mu \mathrm{M})$ was added $30 \mathrm{~min}$ before $\mathrm{FSH}$ treatment.

Transfection: The FoxO1 expression vectors pcDNA3-FLAG-FKHR (WT), pcDNA3-FLAG-FKHR (AAA) and pcDNA3-FLAG-FKHR (F) were kindly provided by Dr. Haojie Huang (University of Minnesota, Minneapolis, MN, USA). pGL3-Basic and PRL-TK were purchased from Promega (Madison, WI, USA). pcDNA3 was obtained from Invitrogen. pGL3-FoxO1 (W) and pGL3-FoxO1 (M) were constructed in our lab. Transfection was performed using Lipofectamine 2000 Reagent (Invitrogen) according to the manufacturer's directions.

Intracellular ROS detection. ROS levels were determined by measuring the oxidative conversion of dihydroethidium bromide to ethidium bromide, which generates red fluorescence when bound to DNA within the cell nuclei. The experiments were conducted using the GENMED Intracellular ROS Red Fluorescence Determination Kit (GENMED, Shanghai, China) according to the manufacturer's directions. Fluorescent images were obtained using a Carl Zeiss laser-scanning confocal microscope (Carl Zeiss, Jena, Germany). The optical density was evaluated in each MGC with ImageJ 1.42q software (National Institutes of Health, Bethesda, MD, USA).

Measurement of caspase activity. Activities of caspases were measured as described previously. ${ }^{65}$ Caspase-3 activity was analyzed using the Caspase-3 Activity Assay Kit (Beyotime) with the fluorogenic substrate Ac-DEVD-pNA (acetylAsp-Glu-Val-Asp p-nitroanilide). Caspase-8 and -9 activities were measured using Ac-IETD-pNA (acetyl-Ile-Glu-Thr-Asp p-nitroanilide, Beyotime) and Ac-LEHD-pNA (acetyl-Leu-Glu-His-Asp p-nitroanilide, Beyotime), respectively. The experimental procedures were performed according to the manufacturer's instructions. Briefly, cells were digested in $0.25 \%$ Trypsin-EDTA (Gibco), pelleted by centrifugation at 3000 r.p.m. for $5 \mathrm{~min}$, and lysed in $50 \mu \mathrm{l}$ lysis buffer for $15 \mathrm{~min}$ on ice. Whole-cell lysates were centrifuged at $20000 \times g$ for $15 \mathrm{~min}$ at $4{ }^{\circ} \mathrm{C}$, and the supernatants were collected. The protein concentration was determined with the Bradford Protein Assay Kit (Beyotime). Ten micrograms of total protein was added to a 96-well plate containing $100 \mu \mathrm{l}$ buffer plus caspase substrates and incubated for $2 \mathrm{~h}$ at $37^{\circ} \mathrm{C}$. Cleavage of the substrates yielded a yellow product, $\mathrm{p}$-nitroaniline, which was detected at $405 \mathrm{~nm}$ using a microplate reader (Bio-Rad, Hercules, CA, USA).

Western blotting. Cells were harvested in RIPA lysis buffer, and protein was quantified with the BCA assay (Beyotime). Whole-cell lysates containing $15 \mu \mathrm{g}$ total protein were loaded onto $7.5 \%$ sodium dodecyl sulfate (SDS)-polyacrylamide gels, separated by electrophoresis, and transferred to polyvinylidene difluoride membranes (Millipore, Billerica, MA, USA). Nonspecific binding on the membranes was blocked with $5 \%$ bovine serum albumin (BSA) in TBS-T ( $50 \mathrm{mM}$ Tris-HCl, pH $7.5,150 \mathrm{mM} \mathrm{NaCl}, 0.1 \%$ Tween 20) for $1 \mathrm{~h}$ at room temperature. Membranes were then probed with primary antibodies $(1: 1000)$ against mouse Fox01, DYKDDDDK Tag (Cell Signaling Technology, Danvers, MA, USA) and $\alpha$-tubulin (Sigma) in blocking solution overnight at $4^{\circ} \mathrm{C}$. After washing with TBS-T, membranes were incubated with horseradish peroxidase-conjugated secondary antibodies (Santa Cruz Biotechnology, Santa Cruz, CA, USA) for $1 \mathrm{~h}$ at room temperature. Protein bands were visualized by exposing to an enhanced chemiluminescence detection system (LAS-4000 imager, Fujjilm, Tokyo, Japan) using the SuperSignal West Pico chemiluminescent substrate (Pierce, Rockford, IL, USA). Densitometry analyses were performed using Image J 1.42q software (National Institutes of Health), and the values for target proteins were normalized to $\alpha$-tubulin as the endogenous control.

qRT-PCR. Total RNA was isolated with TRIZOL reagent (Invitrogen) and reversetranscribed into cDNA using the Reverse Transcription System (Promega) according to the manufacturer's instructions. Real-time PCR was performed with SYBR Premix Ex Taq (TaKaRa Biotechnology (Dalian) Co., Ltd., Otsu, Shiga, Japan) and genespecific primers (see Supplementary Table S1 for primer sequences) on the 7300 Standard Real-Time PCR System (Applied Biosystems, Foster City, CA, USA). Melting curves were analyzed to verify amplification specificity. Expression data were normalized to the amount of $\beta$-actin expressed.

Immunofluorescence experiments. MGCs were seeded on coverslips in 12-well plates. Cells were grown to $90 \%$ confluency in 4 days and then exposed to the treatments described above. After washing in PBS, the cell climbing sheets were fixed with $4 \%$ paraformaldehyde for $1 \mathrm{~h}$, permeabilized using $0.5 \%$ Triton $\mathrm{X}-100$ in PBS for $10 \mathrm{~min}$ at $4{ }^{\circ} \mathrm{C}$ and blocked with $1 \% \mathrm{BSA}$ for $1 \mathrm{~h}$ at room temperature. The coverslips were then incubated with anti-FoxO1 antibody (Cell Signaling Technology; $1: 100)$ for $1 \mathrm{~h}$ at $37^{\circ} \mathrm{C}$. The cells were incubated with Alexa Fluor 488 (green)conjugated goat anti-rabbit lgG (Invitrogen; $1: 200$ ) for $1 \mathrm{~h}$ in the dark. After nuclear staining with DAPI (Invitrogen) for $20 \mathrm{~min}$, the coverslips were washed, mounted on slides and observed under a laser-scanning confocal microscope (Carl Zeiss).

H\&E staining. H\&E staining was performed as previously described ${ }^{66}$ Briefly, at 48,72 and $96 \mathrm{~h}$ after the first FSH injection, ovaries collected from mice primed with or without FSH were fixed with $4 \%$ paraformaldehyde, paraffin-embedded, serially sectioned at $5 \mu \mathrm{m}$ thickness and mounted on glass slides. After deparaffinization and rehydration, sections were stained with H\&E (Nanjing Jianchen Institute of Biological Engineering, Nanjing, China) and examined using dot Slide-digital virtual microscopy (Olympus, Tokyo, Japan).

TUNEL assay. Apoptosis was determined with the TUNEL method as described. ${ }^{4}$ The procedures were performed using the In Situ Cell Death Detection Kit (Roche Applied Science, Mannheim, Germany) according to the manufacturer's instructions. After TUNEL reactions, cell climbing sheets or ovarian sections were mounted with VECTASHIELD Mounting Medium plus DAPI and examined under a laser-scanning confocal microscope (Carl Zeiss).

PKA assay. The enzymatic activities were detected using a GENMED Cellular PKA Activity Colorimetric Quantitative Determination Kit, a GENMED Cellular PI3K Activity Colorimetric Quantitative Determination Kit and a GENMED Cellular PKB/ AKT Activity Colorimetric Quantitative Determination Kit (GENMED) according to the manufacturer's instructions. In brief, cells were cultured in T-75 flasks, removed carefully with a cell scraper (Corning Incorporated Life Sciences, Acton, MA, USA), pelleted by centrifugation at 3000 r.p.m. for 5 min, and lysed with $500 \mu l$ lysis buffer for $30 \mathrm{~min}$ on ice. Cell lysates were then centrifuged at $20000 \times \mathrm{g}$ for $20 \mathrm{~min}$ at $4^{\circ} \mathrm{C}$. Total proteins in the supernatants were quantified with the BCA reagent (Beyotime). Protein $(50 \mu \mathrm{g}$ in $5 \mu \mathrm{l})$ was transferred to a 96-well plate in which each well contained $95 \mu \mathrm{l}$ reaction mixture and incubated for $5 \mathrm{~min}$ at $37^{\circ} \mathrm{C}$. Samples were then read with a microplate reader (Bio-Rad).

Luciferase reporter gene assay. The FoxO1 promoter was amplified with PCR from mouse genomic DNA (bases 78-2125, 5'-UTR of FoxO1) containing a FRE (AGTAAACAAA), cloned into the pGL3-Basic plasmid (Promega) at the Kpnl and BgllI (TaKaRa Biotechnology (Dalian) Co., Ltd.) sites and named pGL3-FoxO1 (W) (see Supplementary Figure S3 for primer sequences). pGL3-FoxO1 (M) was obtained by introducing four mutations into FRE using a Mut Express II Fast Mutagenesis Kit (Vazyme, China). Mouse NIH 3 T3 fibroblasts were seeded in a 12-well plate and grown to $70 \%$ density before transfection with Lipofectamine 2000 
(Invitrogen). Three plasmids were co-transfected in each treatment: (1) $1.6 \mu \mathrm{g}$ expression vector pcDNA3-FLAG-FKHR (WT); (2) $1.6 \mu \mathrm{g}$ reporter construct pGL3FoxO1 (W) or pGL3-FoxO1 (M); and (3) $32 \mathrm{ng}$ control reporter pRL-TK (Promega). After $36 \mathrm{~h}$, cells were processed for reporter assays using the Dual-luciferase Reporter Assay System (Promega) and Modulus Microplate Luminometer (Turner BioSystems, Sunnyvale, CA, USA) according to the manufacturer's instructions. All inserts were verified with sequencing.

ChIP assays. ChIP assays were performed according to the manufacturer's protocols in the Pierce Agarose ChIP Kit (Pierce). Briefly, cells were fixed in 1\% formaldehyde for $10 \mathrm{~min}$ at room temperature. Crosslinking was stopped by adding glycine to a final concentration of $125 \mathrm{mM}$. After washing with cold PBS containing protease inhibitors, cells were lysed in $1 \mathrm{ml}$ SDS lysis buffer (1\% SDS, $10 \mathrm{mM}$ EDTA, $50 \mathrm{mM}$ Tris, $\mathrm{pH}$ 8.1). Chromatin was then sheared with sonication to generate fragments $250-750 \mathrm{bp}$. For each ChIP reaction, $10 \%$ of the chromatin was stored as input, and the rest was processed for immunoprecipitation by incubating with anti-FoxO1 (Cell Signaling Technology) and rabbit lgG (as control). Proteinase $\mathrm{K}$ was used to degrade proteins in the precipitated complexes, and DNA was isolated and used as a template for qRT-PCR using the primers indicated in Supplementary Table S1. qRT-PCR products were then electrophoresed on a $2 \%$ agarose gel. The amount of immunoprecipitated DNA for each ChIP reaction is presented as the percent of input chromatin.

Statistical analysis. Data were analyzed using SPSS version 16.0 (SPSS Inc., Chicago, IL, USA). Statistical significance was calculated with the Student's $t$-test $(P<0.05$ was considered statistically significant). All experiments were repeated at least three times. Results were expressed as the mean \pm S.E.

\section{Conflict of Interest}

The authors declare no conflict of interest.

Acknowledgements. We thank Dr. Xiaolong Zhou, Dr Jiying Liu, Ms Peng Xiao and Ms. Rui Chao for their technical support, Dr Mei Li for helpful suggestions during the experimental design, $\mathrm{Dr}$ Haojie Huang for generously providing the FoxO1 expression vectors pcDNA3-FLAG-FKHR (WT), pcDNA3-FLAG-FKHR (AAA) and pcDNA3-FLAG-FKHR (F), and Dr Fei Lin and Ms Xuehai Du for their early contributions to the project. We are also grateful to all the members of the Animal Genetics, Breeding and Reproduction unit for constant assistance. This research was supported by the key Project of Chinese National Programs for Fundamental Research and Development (973 program no. 2014CB138502).

1. Baker TG. A quantitative and cytological study of germ cells in human ovaries. Proc $R$ Soc Lond B Biol Sci 1963; 158: 417-433.

2. Cataldo NA, Dumesic DA, Goldsmith PC, Jaffe RB. Immunolocalization of Fas and Fas ligand in the ovaries of women with polycystic ovary syndrome: relationship to apoptosis. Hum Reprod 2000; 15: 1889-1897.

3. Persani L, Rossetti R, Cacciatore $C$. Genes involved in human premature ovarian failure. $J$ Mol Endocrinol 2010; 45: 257-279.

4. Shen M, Lin F, Zhang J, Tang Y, Chen WK, Liu H. Involvement of the up-regulated FoxO1 expression in follicular granulosa cell apoptosis induced by oxidative stress. J Biol Chem 2012; 287: 25727-25740

5. Kreeger PK, Fernandes NN, Woodruff TK, Shea LD. Regulation of mouse follicle development by follicle-stimulating hormone in a three-dimensional in vitro culture system is dependent on follicle stage and dose. Biol Reprod 2005; 73: 942-950.

6. Asselin E, Xiao CW, Wang YF, Tsang BK. Mammalian follicular development and atresia: role of apoptosis. Biol Signals Recept 2000; 9: 87-95.

7. Dorrington JH, Moon YS, Armstrong DT. Estradiol-17beta biosynthesis in cultured granulosa cells from hypophysectomized immature rats; stimulation by follicle-stimulating hormone. Endocrinology 1975; 97: 1328-1331.

8. Kumar TR, Wang Y, Lu N, Matzuk MM. Follicle stimulating hormone is required for ovarian follicle maturation but not male fertility. Nat Genet 1997; 15: 201-204.

9. Fauser BC, Van Heusden AM. Manipulation of human ovarian function: physiological concepts and clinical consequences. Endocr Rev 1997; 18: 71-106.

10. Hunzicker-Dunn M, Maizels ET. FSH signaling pathways in immature granulosa cells that regulate target gene expression: branching out from protein kinase A. Cell Signal 2006; 18 : 1351-1359.

11. Oxberry BA, Greenwald GS. An autoradiographic study of the binding of 125 I-labeled follicle-stimulating hormone, human chorionic gonadotropin and prolactin to the hamster ovary throughout the estrous cycle. Biol Reprod 1982; 27: 505-516.
12. Chun SY, Eisenhauer KM, Minami S, Billig H, Perlas E, Hsueh AJ. Hormonal regulation of apoptosis in early antral follicles: follicle-stimulating hormone as a major survival factor. Endocrinology 1996; 137: 1447-1456.

13. Wang XL, Wu Y, Tan LB, Tian Z, Liu JH, Zhu DS et al. Follicle-stimulating hormone regulates pro-apoptotic protein Bcl-2-interacting mediator of cell death-extra long (BimEL)-induced porcine granulosa cell apoptosis. J Biol Chem 2012; 287: 10166-10177.

14. Sanchez AM, Candau RB, Bernardi H. FoxO transcription factors: their roles in the maintenance of skeletal muscle homeostasis. Cell Mol Life Sci 2014; 71: 1657-1671.

15. Huang H, Tindall DJ. Dynamic FoxO transcription factors. J Cell Sci 2007; 120: 2479-2487.

16. Nakamura N, Ramaswamy S, Vazquez F, Signoretti S, Loda M, Sellers WR. Forkhead transcription factors are critical effectors of cell death and cell cycle arrest downstream of PTEN. Mol Cell Biol 2000; 20: 8969-8982.

17. Dijkers PF, Medema RH, Pals C, Banerji L, Thomas NS, Lam EW et al. Forkhead transcription factor FKHR-L1 modulates cytokine-dependent transcriptional regulation of p27 (KIP1). Mol Cell Biol 2000; 20: 9138-9148.

18. Gilley J, Coffer PJ, Ham J. FOXO transcription factors directly activate bim gene expression and promote apoptosis in sympathetic neurons. J Cell Biol 2003; 162: 613-622.

19. Modur V, Nagarajan R, Evers BM, Milbrandt J. FOXO proteins regulate tumor necrosis factor-related apoptosis inducing ligand expression. Implications for PTEN mutation in prostate cancer. J Biol Chem 2002; 277: 47928-47937.

20. Lee SS, Kennedy S, Tolonen AC, Ruvkun G. DAF-16 target genes that control C. elegans lifespan and metabolism. Science 2003; 300: 644-647.

21. Greer EL, Brunet A. FOXO transcription factors in ageing and cancer. Acta Physiol (Oxf) 2008; 192: 19-28.

22. Liu Z, Rudd MD, Hernandez-Gonzalez I, Gonzalez-Robayna I, Fan HY, Zeleznik AJ et al. FSH and FOXO1 regulate genes in the sterol/steroid and lipid biosynthetic pathways in granulosa cells. Mol Endocrinol 2009; 23: 649-661.

23. Shi F, LaPolt PS. Relationship between Fox01 protein levels and follicular development, atresia, and luteinization in the rat ovary. J Endocrinol 2003; 179: 195-203.

24. Fan HY, O'Connor A, Shitanaka M, Shimada M, Liu Z, Richards JS. Beta-catenin (CTNNB1) promotes preovulatory follicular development but represses LH-mediated ovulation and luteinization. Mol Endocrinol 2010; 24: 1529-1542.

25. Park Y, Maizels ET, Feiger ZJ, Alam H, Peters CA, Woodruff TK et al. Induction of cyclin D2 in rat granulosa cells requires FSH-dependent relief from FOXO1 repression coupled with positive signals from Smad. J Biol Chem 2005; 280: 9135-9148.

26. Rosairo D, Kuyznierewicz I, Findlay J, Drummond A. Transforming growth factor-beta: its role in ovarian follicle development. Reproduction 2008; 136: 799-809.

27. Li X, Jiang Y, Wang Z, Liu G, Hutz RJ, Liu W et al. Regulation of FoxO1 transcription factor by nitric oxide and cyclic GMP in cultured rat granulosa cells. Zoolog Sci 2005; 22: 1339-1346.

28. Maillet G. Feral C, Benhaim A. [Apoptosis of the follicular cells: its implication in ovarian induction protocols]. Gynecol Obstet Fertil 2005; 33: 653-658.

29. Markstrom E, Svensson E, Shao R, Svanberg B, Billig H. Survival factors regulating ovarian apoptosis - dependence on follicle differentiation. Reproduction 2002; 123: 23-30.

30. Peluso JJ, Steger RW. Role of FSH in regulating granulosa cell division and follicular atresia in rats. J Reprod Fertil 1978; 54: 275-278.

31. Matsuda-Minehata F, Inoue N, Goto Y, Manabe N. The regulation of ovarian granulosa cell death by pro- and anti-apoptotic molecules. J Reprod Dev 2006; 52: 695-705.

32. Zhou XL, Teng Y, Cao R, Fu H, Xiong K, Sun WX et al. Rescue from dominant follicle atresia by follicle-stimulating hormone in mice. Genet Mol Res 2013; 12: 2945-2952.

33. Liu Z, Castrillon DH, Zhou W, Richards JS. FOXO1/3 depletion in granulosa cells alters follicle growth, death and regulation of pituitary FSH. Mol Endocrinol 2013; 27 : 238-252.

34. Harris $\mathrm{CA}$, Johnson $\mathrm{EM} \mathrm{Jr}$. BH3-only $\mathrm{Bcl}-2$ family members are coordinately regulated by the JNK pathway and require Bax to induce apoptosis in neurons. J Biol Chem 2001; 276: 37754-37760.

35. Wang XN, Greenwald GS. Synergistic effects of steroids with FSH on folliculogenesis, steroidogenesis and FSH- and hCG-receptors in hypophysectomized mice. J Reprod Fertil 1993; 99: 403-413

36. Al-Mubarak B, Soriano FX, Hardingham GE. Synaptic NMDAR activity suppresses FOXO1 expression via a cis-acting FOXO binding site: FOXO1 is a FOXO target gene. Channels (Austin) 2009; 3: 233-238.

37. Essaghir A, Dif N, Marbehant CY, Coffer PJ, Demoulin JB. The transcription of FOXO genes is stimulated by FOXO3 and repressed by growth factors. J Biol Chem 2009; 284: 10334-10342.

38. Zhou J, Liao W, Yang J, Ma K, Li X, Wang Y et al. FOXO3 induces FOXO1-dependent autophagy by activating the AKT1 signaling pathway. Autophagy 2012; 8: 1712-1723.

39. Richards JS, Sharma SC, Falender AE, Lo YH. Expression of FKHR, FKHRL1, and AFX genes in the rodent ovary: evidence for regulation by IGF-I, estrogen, and the gonadotropins. Mol Endocrinol 2002; 16: 580-599.

40. Burgering BM, Medema RH. Decisions on life and death: FOXO Forkhead transcription factors are in command when PKB/Akt is off duty. J Leukoc Biol 2003; 73: 689-701.

41. Webb R, Campbell BK. Development of the dominant follicle: mechanisms of selection and maintenance of oocyte quality. Soc Reprod Fertil Suppl 2007; 64: 141-163.

42. Dhanasekaran N, Moudgal NR. Studies on follicular atresia: role of tropic hormone and steroids in regulating cathepsin-D activity of preantral follicles of the immature rat. Mol Cell Endocrinol 1986; 44: 77-84. 
43. Parborell F, Dain L, Tesone M. Gonadotropin-releasing hormone agonist affects rat ovarian follicle development by interfering with $\mathrm{FSH}$ and growth factors on the prevention of apoptosis. Mol Reprod Dev 2001; 60: 241-247.

44. Chun SY, Billig H, Tilly JL, Furuta I, Tsafriri A, Hsueh AJ. Gonadotropin suppression of apoptosis in cultured preovulatory follicles: mediatory role of endogenous insulin-like growth factor I. Endocrinology 1994; 135: 1845-1853.

45. Cunningham MA, Zhu Q, Unterman TG, Hammond JM. Follicle-stimulating hormone promotes nuclear exclusion of the forkhead transcription factor FoxO1a via phosphatidylinositol 3-kinase in porcine granulosa cells. Endocrinology 2003; 144: 5585-5594.

46. Earnshaw WC, Martins LM, Kaufmann SH. Mammalian caspases: structure, activation, substrates, and functions during apoptosis. Annu Rev Biochem 1999; 68: 383-424.

47. Hsu SY, Lin P, Hsueh AJ. BOD (Bcl-2-related ovarian death gene) is an ovarian $\mathrm{BH} 3$ domain-containing proapoptotic $\mathrm{Bcl}-2$ protein capable of dimerization with diverse antiapoptotic Bcl-2 members. Mol Endocrinol 1998; 12: 1432-1440.

48. Brunet A, Bonni A, Zigmond MJ, Lin MZ, Juo P, Hu LS et al. Akt promotes cell survival by phosphorylating and inhibiting a Forkhead transcription factor. Cell 1999; 96: 857-868.

49. Urbich C, Knau A, Fichtlscherer S, Walter DH, Bruhl T, Potente M et al. FOXO-dependent expression of the proapoptotic protein Bim: pivotal role for apoptosis signaling in endothelial progenitor cells. FASEB J 2005; 19: 974-976.

50. Adachi M, Osawa $Y$, Uchinami $H$, Kitamura $T$, Accili D, Brenner DA. The forkhead transcription factor FoxO1 regulates proliferation and transdifferentiation of hepatic stellate cells. Gastroenterology 2007; 132: 1434-1446.

51. Carr DW, Cutler Jr RE, Cottom JE, Salvador LM, Fraser ID, Scott JD et al. Identification of CAMP-dependent protein kinase holoenzymes in preantral- and preovulatory-follicleenriched ovaries, and their association with A-kinase-anchoring proteins. Biochem J 1999; 344(Pt 2): 613-623.

52. Alam H, Maizels ET, Park Y, Ghaey S, Feiger ZJ, Chandel NS et al. Follicle-stimulating hormone activation of hypoxia-inducible factor-1 by the phosphatidylinositol 3-kinase/AKT/ Ras homolog enriched in brain (Rheb)/mammalian target of rapamycin (mTOR) pathway is necessary for induction of select protein markers of follicular differentiation. $J$ Biol Chem 2004; 279: 19431-19440.

53. Gonzalez-Robayna IJ, Falender AE, Ochsner S, Firestone GL, Richards JS Follicle-stimulating hormone (FSH) stimulates phosphorylation and activation of protein kinase $B(P K B / A k t)$ and serum and glucocorticoid-Induced kinase (Sgk): evidence for A kinase-independent signaling by FSH in granulosa cells. Mol Endocrinol 2000; 14 1283-1300.

54. Roy SK, Srivastava RK, Shankar S. Inhibition of PI3K/AKT and MAPK/ERK pathways causes activation of FOXO transcription factor, leading to cell cycle arrest and apoptosis in pancreatic cancer. J Mol Signal 2010; 5: 10.

55. Chang L, Karin M. Mammalian MAP kinase signalling cascades. Nature 2001; 410: 37-40.

56. Cottom J, Salvador LM, Maizels ET, Reierstad S, Park Y, Carr DW et al. Follicle-stimulating hormone activates extracellular signal-regulated kinase but not extracellular signal-regulated kinase kinase through a 100-kDa phosphotyrosine phosphatase. J Biol Chem 2003; 278 7167-7179.

57. Nakae J, Barr V, Accili D. Differential regulation of gene expression by insulin and IGF-1 receptors correlates with phosphorylation of a single amino acid residue in the forkhead transcription factor FKHR. EMBO J 2000; 19: 989-996.

58. Lutzner $\mathrm{N}$, Kalbacher $\mathrm{H}$, Krones-Herzig A, RosI F. FOXO3 is a glucocorticoid receptor target and regulates LKB1 and its own expression based on cellular AMP levels via a positive autoregulatory loop. PLoS One 2012; 7: e42166.

59. Kuiper GG, Enmark E, Pelto-Huikko M, Nilsson S, Gustafsson JA. Cloning of a novel receptor expressed in rat prostate and ovary. Proc Natl Acad Sci USA 1996; 93: $5925-5930$.

60. Munagala R, Aqil F, Vadhanam MV, Gupta RC. MicroRNA 'signature' during estrogenmediated mammary carcinogenesis and its reversal by ellagic acid intervention. Cancer Lett 2013; 339: 175-184.

61. Foulds CE, Feng Q, Ding C, Bailey S, Hunsaker TL, Malovannaya A et al. Proteomic analysis of coregulators bound to ERalpha on DNA and nucleosomes reveals coregulator dynamics. Mol Cell 2013; 51: 185-199.

62. Lombardi M, Castoria G, Migliaccio A, Barone MV, Di Stasio R, Ciociola A et al. Hormone-dependent nuclear export of estradiol receptor and DNA synthesis in breast cancer cells. J Cell Biol 2008; 182: 327-340.

63. Nicolson GL, Yanagimachi R, Yanagimachi H. Ultrastructural localization of lectin-binding sites on the zonae pellucidae and plasma membranes of mammalian eggs. J Cell Biol 1975 66: 263-274.

64. Luo XG, Xi T, Guo S, Liu ZP, Wang N, Jiang Y et al. Effects of SMYD3 overexpression on transformation, serum dependence, and apoptosis sensitivity in NIH3T3 cells. IUBMB Life 2009; 61: 679-684.

65. Liu C, Yu K, Shi X, Wang J, Lam PK, Wu RS et al. Induction of oxidative stress and apoptosis by PFOS and PFOA in primary cultured hepatocytes of freshwater tilapia (Oreochromis niloticus). Aquat Toxicol 2007; 82: 135-143.

66. Zhang JQ, Shen M, Zhu CC, Yu FX, Liu ZQ, Ally N et al. 3-Nitropropionic acid induces ovarian oxidative stress and impairs follicle in mouse. PLOS One 2014; 9: e86589.

(c) Cell Death and Disease is an open-access journal published by Nature Publishing Group. This work is licensed under a Creative Commons Attribution 4.0 International Licence. The images or other third party material in this article are included in the article's Creative Commons licence, unless indicated otherwise in the credit line; if the material is not included under the Creative Commons licence, users will need to obtain permission from the licence holder to reproduce the material. To view a copy of this licence, visit http://creativecommons.org/licenses/by/4.0

Supplementary Information accompanies this paper on Cell Death and Disease website (http://www.nature.com/cddis) 